\title{
Ketogenic Diet Improves Brain Ischemic Tolerance and Inhibits NLRP3 Inflammasome Activation by Preventing Drp1-Mediated Mitochondrial Fission and Endoplasmic Reticulum Stress
}

\author{
Min Guo ${ }^{1 \dagger}$, Xun Wang ${ }^{1 \dagger}$, Yanxin Zhao ${ }^{2}$, Qi Yang ${ }^{1}$, Hongyan Ding ${ }^{1}$, Qiang Dong ${ }^{1}$, \\ Xingdong Chen ${ }^{3,4 *}$ and Mei Cui ${ }^{1 *}$
}

${ }^{1}$ Department of Neurology, Huashan Hospital, Fudan University, Shanghai, China, ${ }^{2}$ Department of Neurology, Shanghai Tenth People's Hospital, Tongji University, Shanghai, China, ${ }^{3}$ The State Key Laboratory of Genetic Engineering, Collaborative Innovativation Center for Genetics and Development, School of Life Science, Fudan University, Shanghai, China, ${ }^{4}$ Fudan University Taizhou Institute of Health Science, Taizhou, China

OPEN ACCESS

Edited by:

Andrei Surguchov, University of Kansas Medical Center, United States

Reviewed by: Mustapha Umar Imam, Zhengzhou University, China David Ruskin, Trinity College, United States

*Correspondence: Xingdong Chen xingdongchen@fudan.edu.cn Mei Cui cuimei@fudan.edu.cn

${ }^{\dagger}$ These authors have contributed equally to this work.

Received: 29 November 2017 Accepted: 05 March 2018

Published: 20 March 2018

Citation:

Guo M, Wang X, Zhao Y, Yang Q, Ding $H$, Dong $Q$, Chen $X$ and Cui $M$ (2018) Ketogenic Diet Improves Brain Ischemic Tolerance and Inhibits NLRP3 Inflammasome Activation by Preventing Drp1-Mediated Mitochondrial Fission and Endoplasmic Reticulum Stress. Front. Mol. Neurosci. 11:86. doi: 10.3389/fnmol.2018.00086
Background: Neuroprotective effects of ketogenic diets (KD) have been reported in stroke models, and nucleotide-binding domain (NOD)-like receptor protein 3 (NLRP3) inflammasome has also been implicated in the pathogenesis of stroke. This study aimed to investigate the effects of KD on NLRP3 inflammasome and explore the potential molecular mechanisms.

Methods: In in vivo study, mice were fed with KD for 3 weeks and then subjected to middle cerebral artery occlusion/reperfusion (MCAO/R)-injury. In in vitro study, SH-SY-5Y cells were treated with $\beta$-hydroxybutyrate (BHB) followed by oxygen-glucose deprivation/reoxygenation (OGD/R). NLRP3 inflammasome activation and related regulatory mechanisms were evaluated.

Results: Mice fed with $\mathrm{KD}$ had increased tolerance to MCAO/R. KD inhibited endoplasmic reticulum (ER) stress and suppressed TXNIP/NLRP3 inflammasome activation in the brain. The in vitro study showed BHB $(10 \mathrm{mM})$ prevented the mitochondrial translocation of dynamin-related protein 1 (Drp1) to inhibit mitochondrial fission. Furthermore, BHB decreased reactive oxygen species (ROS) generation, inhibited ROS-NLRP3 pathway in OGD/R-treated cells, and suppressed ER stressinduced NLRP3 inflammasome activation.

Conclusions: KD may suppress ER stress and protect mitochondrial integrity by suppressing the mitochondrial translocation of Drp1 to inhibit NLRP3 inflammasome

\footnotetext{
Abbreviations: BBB, blood-brain barrier; BHB, b-hydroxybutyrate; Drp1, dynamin-related protein 1; IL-1 $\beta$, interleukin-1 $\beta$; KBs, ketone bodies; KD, ketogenic diets; $\mathrm{MCAO} / \mathrm{R}$, middle cerebral artery occlusion/reperfusion; MMP, mitochondrial membrane potential; NLRP3, nucleotide-binding domain (NOD)-like receptor protein 3; OGD/R, oxygen-glucose deprivation/reoxygenation; ROS, reactive oxygen species; TTC, 2,3,5-triphenyltetrazolium chloride; TXNIP, thioredoxininteracting protein; UPR, unfolded protein response.
} 
activation, thus exerting neuroprotective effects. Our findings provide evidence for the potential application of KD in the prevention of ischemic stroke.

Keywords: ketogenic diet, $\beta$-hydroxybutyrate, mitochondrial fission, endoplasmic reticulum stress, NLRP3 inflammasome, Drp1

\section{INTRODUCTION}

Ischemic stroke, a leading cause of destructive cerebrovascular diseases, is characterized by disrupted blood flow and glucose/oxygen deprivation of brain cells, which may result in cellular dysfunction (Fisher and Saver, 2015). Current treatments for ischemic stroke are limited because of its complex molecular mechanisms (Barrett et al., 2015). Inflammation plays an important role in the pathogenesis of ischemic stroke, and the role of nucleotide-binding domain (NOD)-like receptor protein 3 (NLRP3) inflammasome in stroke has been a focus in current studies (Kawabori and Yenari, 2015). The NLRP3 inflammasome is a molecular platform in which pro-inflammatory cytokines (such as caspase-1 and interleukin-1 $\beta$ [IL-1 $\beta$ ]) are activated to induce inflammation (Ogura et al., 2006). It has been confirmed that NLRP3 inflammasome is activated since the onset of ischemic stroke (Fann et al., 2013b), and treatments targeting NLRP3 inflammasome are found to be promising for the treatment of stroke (Yang et al., 2014).

Under physiological condition, NLRP3 typically localizes in the endoplasmic reticulum (ER). Upon activation, NLRP3 and its adaptor apoptosis-associated Speck-like protein (ASC) translocate to the perinuclear space, where they co-localize with ER and mitochondrial organelle clusters (Jo et al., 2016). Mitochondria are remarkably dynamic organelles that can traffic, divide and fuse. The cycles of mitochondrial fission and fusion ensure normal metabolic and bioenergetic functions (Bertholet et al., 2016). Mitochondrial fission can trigger the NLRP3 inflammasome upon RNA virus infection, in which, dynamin-related protein1 (Drp1), a key regulator of mitochondrial fission, plays an important role (Rayamajhi and Miao, 2014). Our previous study revealed that Drp1 translocating to the mitochondria mediated the mitochondrial fission, and pharmacological inhibition of Drp1 translocation prevented mitochondrial fragmentation, therefore protecting neurons from oxygen-glucose deprivation (OGD) induced injury (Zhao et al., 2013). However, the mechanism by which Drp1 regulates NLRP3-mediated inflammation in ischemic stroke remains unclear.

Following ischemia, the newly synthesized proteins accumulate to increase the unfolded protein response (UPR). Severe and prolonged UPR may induce ER stress. The ER stress sensor IRE1a can induce thioredoxin-interacting protein (TXNIP) to activate NLRP3-mediated inflammation and programmed cell death (Lerner et al., 2012). Reactive oxygen species (ROS) generated from mitochondrial fission can activate NLRP3 inflammasome to exacerbate ER stress, which further impairs the mitochondrial morphology and function (Minutoli et al., 2016). Suppression of ER stress and/or ROS generation has also been found to alleviate NLRP3-mediated inflammation in stroke.
Disruption of blood flow and/or a decrease in blood glucose concentration in ischemic stroke may cause brain injury. Ketone bodies (KBs), which include acetoacetate and $\beta$-hydroxybutyrate (BHB), may serve as alternative substrates to glucose in the brain. $\mathrm{BHB}$ is a major member of KBs. It is generated from fatty acid oxidation and can be converted into BHB by ketogenesis in the liver mitochondria. A ketogenic diet (KD) or BHB has been found to improve the cerebral edema and infarct volume following stroke (Suzuki et al., 2001). Moreover, BHB can prevent neuronal death induced by glucose deprivation or hypoxia (Camberos-Luna et al., 2016). Recent studies also reveal that $\mathrm{BHB}$ is able to suppress the NLRP3 inflammasome activation in human hepatoma HepG2 cells and monocytes (Youm et al., 2015; Bae et al., 2016). However, the role of KBs in the regulation of NLRP3 inflammasome in the central nervous system after ischemia remains unknown.

In this study, effects of $\mathrm{KD}$ and $\mathrm{BHB}$ on NLRP3 inflammasome were investigated in mice with middle cerebral artery occlusion (MCAO) and SH-SY-5Y cells subjected to $\mathrm{OGD} /$ reoxygenation (OGD/R), respectively, and the potential mechanism was further explored. Our results revealed that $\mathrm{KD}$ and $\mathrm{BHB}$ were able to improve cerebral ischemia by inhibiting NLRP3 inflammasome activation, which was ascribed to the suppression of Drp1-mediated mitochondrial fission and the inhibition of ER stress.

\section{MATERIALS AND METHODS}

\section{Animals and Dietary Protocols}

Male C57BL/6 mice (4 weeks of age) were housed in the Experimental Animal Center of Fudan University at $26^{\circ} \mathrm{C}$ with $12 \mathrm{~h} / 12 \mathrm{~h}$ light/dark cycles and given ad libitum access to food and water. Mice were randomly assigned to four groups: control group (mice were fed with standard chow [Teklab, 8664]); KD group (mice were fed with high fat, low carbohydrate diet [ResearchDiets, D12369B]); high carbohydrate (HC) diet group (mice were fed with low fat, HC diet [ResearchDiets, D12359]); CP-456773 group (mice received a single dose of oral CP-456773 [50 mg/kg, NLRP3 inflammasome inhibitor, Sigma PZ0280] for 3 weeks with standard chow). Mice were fed with assigned diets (the composition of three diets is shown in Table 1) for 3 weeks. Prior to randomization, mice in each group were fasted for $18 \mathrm{~h}$ to stabilize the blood glucose level and to initiate a state of ketosis. All procedures were performed in accordance with the Guide for the National Science Council of the People's Republic of China. The study was approved by the Ethics Committee of Fudan University, Shanghai, China. The approval number from IRB is "20150572A259". This manuscript was written in accordance with the Animal Research: Reporting in vivo Experiments (ARRIVE) guideline. 
TABLE 1 | Macronutrient composition of mouse diets used in this study.

\begin{tabular}{lccc}
\hline Diet & Fat (\%)* & Protein (\%)* & Carb (\%)* \\
\hline Ketogenic diet (KD) & 89.5 & 10.4 & 0.1 \\
High carbohydrate diet (HC) & 11.5 & 10.4 & 78.1 \\
Standard lab-chow (Control) & 27.5 & 20.0 & 52.6 \\
\hline
\end{tabular}

All diets are sterilized before feeding. *Values represent percentage of total calorie.

\section{Establishment of the MCAO Model}

Mice were anesthetized by intraperitoneal injection of ketamine at $65 \mathrm{mg} / \mathrm{kg}$ and xylazine at $6 \mathrm{mg} / \mathrm{kg}$. A coated filament was inserted into the right middle cerebral artery (MCA) for 45-min occlusion, followed by reperfusion. The body temperature was maintained at $37^{\circ} \mathrm{C}$ by using a heating pad and feedback control system (Bowdoin, ME, USA) during the operation. A laser Doppler probe was placed on the skull ( $5 \mathrm{~mm}$ lateral and $2 \mathrm{~mm}$ posterior to the bregma) to monitor the cerebral blood flow (CBF). Mice with $<25 \% \mathrm{CBF}$ reduction after occlusion and $\sim 80 \%$ CBF increase upon reperfusion were included for further analysis. The sham-operated mice underwent the same operation but without ischemia.

\section{Assessment of Infarct Volume, Neurological Deficits and Blood-Brain Barrier}

Infarct volume was determined after 72 -h reperfusion. Brains were harvested and sliced, followed by incubation for $1 \mathrm{~h}$ with 2,3,5-triphenyltetrazolium chloride (TTC). The infarct area was white, while the intact area was red. The infarct area was determined by measuring the total contralateral hemisphere and subtracting the normal tissue area on the ipsilateral hemisphere using Image Pro Plus 6.0 (Doerfler et al., 2001).

Neurological score was determined after 72 -h reperfusion as follows: 0 = no deficit; 1 = forelimb weakness and torso turning to the ipsilateral side when held by tail; $2=$ circling to the affected side; 3 = unable to bear weight on affected side; $4=$ no spontaneous locomotor activity or barrel rolling.

Blood-brain barrier (BBB) permeability was measured after Evans Blue injection. Evans Blue (Sigma-Aldrich) was dissolved in $2 \%$ saline and injected into the right jugular vein after $72-\mathrm{h}$ reperfusion $(0.2 \mathrm{ml} / \mathrm{kg})$. Evans blue was allowed to circulate in bloodstream for $2 \mathrm{~h}$ before mice receiving transcardially perfusion with PBS. Then, animals were sacrificed, and the brains were homogenized in $3 \mathrm{ml}$ of $\mathrm{N}, \mathrm{N}$-dimethylformamide (Sigma-Aldrich), followed by incubation at $55^{\circ} \mathrm{C}$ for $18 \mathrm{~h}$. After centrifugation at $12,000 \mathrm{~g}$ for $20 \mathrm{~min}$, the concentration of Evans Blue in the supernatant was determined by measuring the absorbance at $610 \mathrm{~nm}$ by spectrophotometry.

Investigators blinded to grouping and treatments evaluated the infarct volume and neurological score.

\section{Measurement of Plasma BHB}

Plasma BHB levels were detected by using an enzymatic assay kit (ColorimetricAssay Kit, cat\# K632-100, BioVision Inc., Milpitas, CA, USA). On the last day of week 3, blood samples were collected from the tail of each mouse. To remove interfering substance from the serum, the sample was spun filtered $(10 \mathrm{kDa}$
MWCO spin filter, BioVision, cat\#1997-25). Briefly, $50 \mu \mathrm{l}$ of filtered serum was mixed with $50 \mu \mathrm{l}$ of reaction mix (46 $\mu \mathrm{l} \mathrm{BHB}$ assay buffer, $2 \mu \mathrm{l}$ of BHB enzyme mix and $2 \mu \mathrm{l}$ of substrate mix). The mixture was carefully mixed in a microtiter plate, and then incubated at room temperature for $30 \mathrm{~min}$, and OD value was measured at $450 \mathrm{~nm}$ in a Microplate Reader (BioTek Synergy H1).

\section{TUNEL Staining}

Animals were sacrificed after $72 \mathrm{~h}$ reperfusion, and brain sections (5 $\mu \mathrm{m})$ were obtained and processed for TUNEL staining with the Apop Tag kit (Intergen, Purchase, NY, USA). Briefly, brain sections were fixed in $4 \%$ paraformaldehyde for $5 \mathrm{~min}$, washed twice with PBS, and incubated in permeabilization solution ( $0.1 \%$ Triton X-100 and $0.1 \%$ sodium citrate) for $30 \mathrm{~min}$ at $70^{\circ} \mathrm{C}$. Subsequently, the sections were washed twice in PBS and incubated for $60 \mathrm{~min}$ at $37^{\circ} \mathrm{C}$ in "TUNEL reaction mix". After washing in PBS twice, sections were incubated in DAPI (Molecular Probes, Eugene, OR, USA; 1:1000) for 10 min. Finally, these sections were rinsed with distilled water and treated with anti-fade mounting medium. TUNEL-positive cells were counted from 20 fields $(200 \times$, four fields per section; BioQuant software, Oxford, UK) in each mouse.

\section{Cell Culture and Drug Treatment in Vitro}

SH-SY-5Y cells (CRL-2266, ATCC) were cultured in DMEM-F12 (Sigma D8437) containing 10\% fetal bovine serum (FBS) and antibiotics $(100 \mathrm{U} / \mathrm{ml}$ penicillin $\mathrm{G}$ and $100 \mu \mathrm{g} / \mathrm{ml}$ streptomycin sulfate) at $37^{\circ} \mathrm{C}$ in a humidified atmosphere with $5 \% \mathrm{CO}_{2}$. Twenty-four hours after seeding, differentiation was induced by lowering the FBS in culture medium to $1 \%$ with $10 \mu \mathrm{M}$ retinoic-acid (Sigma-Aldrich, R2625) for 7 days prior to treatment. Cell morphology were evaluated under phase contrast light microscopy and culture medium was replaced once every 2 days to replenish retinoic-acid. Then at $80 \%$ confluence, cells were either treated with $\mathrm{BHB}(10 \mu \mathrm{M}$, OGD+BHB group) or not treated (OGD group), and then subjected to OGD/R (hypoxia $\left[<0.1 \% \mathrm{O}_{2}, 95 \% \mathrm{~N}_{2}, 5 \% \mathrm{CO}_{2}\right]$ and glucose-free media at $37^{\circ} \mathrm{C}$ for $6 \mathrm{~h}$, followed by reoxygenation for $1 \mathrm{~h}$ ). Cells in BHB group were treated with $\mathrm{BHB}(10 \mu \mathrm{M})$ only and cells without any treatment served as control (Ctrl group). For drug treatment, cells were primed with mitochondrial division Drp (dynaminrelated GTPase) inhibitor Mdivi-1 (10 $\mu \mathrm{M}$, Sigma M0199, OGD+MDV group), ER stress inhibitor tauroursodeoxycholic acid (TUDCA, $100 \mu \mathrm{M}$, Sigma T0266, OGD+TUDCA group) or ROS inhibitor N-Acetyl-L-cysteine (NAC, $5 \mathrm{mM}$, Sigma A9165, OGD+NAC group) for $30 \mathrm{~min}$, and subsequently subjected to OGD/R. Specially, cells were treated with ER stress agonist tunicamycin $(2 \mu \mathrm{g} / \mathrm{ml}$, Sigma T7765, Tu group) for $24 \mathrm{~h}$ to induce ER stress prior to $\mathrm{OGD} / \mathrm{R}$.

\section{Lactate Dehydrogenase (LDH) and MTT Assays}

Cell injury was determined by lactate dehydrogenase (LDH) assay with the CytoTox 96 NonRadioactive Cytotoxicity Assay kit (Promega, Madison, WI, USA) according to the manufacturer's instructions. Experiment was done in triplicate. SH-SY-5Y cells 
were cultured in a 96-well culture plate and treated with drugs or OGD/R. At the end of the treatment, $50 \mu \mathrm{l}$ of supernatant was transferred to each well of the 96-well plate, followed by addition of $50 \mu \mathrm{l}$ of reconstituted substrate mix to each well. The plate was incubated at room temperature for $30 \mathrm{~min}$ in dark. After $50 \mu \mathrm{l}$ of stop solution was added to each well, the absorbance was measured at $490 \mathrm{~nm}$. LDH content of the conditional medium was expressed as a percentage of maximal LDH release, after the subtraction of background determined from the medium alone.

MTT assay was employed to determine the cell viability. SH-SY-5Y cells were seeded into a 96-well plate $\left(5 \times 10^{3}\right.$ cells/well). In brief, $20 \mu \mathrm{l}$ of MTT solution $(5 \mathrm{mg} / \mathrm{ml})$ was added to each well at a final concentration of $0.5 \mathrm{mg} / \mathrm{mL}$, followed by incubation for $4 \mathrm{~h}$. The supernatant was removed, and $150 \mu \mathrm{L}$ of dimethylsulfoxide solution was added to each well, followed by incubation for $20 \mathrm{~min}$. The absorbance was measured at $570 \mathrm{~nm}$ with a reference wavelength at $630 \mathrm{~nm}$. Experiment was done in triplicate.

\section{Measurement of ATP}

ATP generation was detected by high performance liquid chromatography (HPLC) as previously reported (Cui et al., 2010). Briefly, the culture medium was removed from the cells, and liquid nitrogen was immediately added. After evaporation on ice, $300 \mu \mathrm{L}$ of ice-cold $0.4 \mathrm{M}$ perchloric acid was added to each well. Cells were collected, and centrifuged at 14,000 $\mathrm{g}$ for $15 \mathrm{~min}$ at $4^{\circ} \mathrm{C}$. The supernatant was neutralized with $1 \mathrm{M}$ $\mathrm{K}_{2} \mathrm{CO}_{3}$, maintained at $-80^{\circ} \mathrm{C}$ to precipitate the perchlorate, and then centrifuged. The supernatant was collected for HPLC. A 12-channel CoulArray 5600A (ESA Inc.) and a reversephase column (Lichrospher-100, Merck) were used. Signals were detected using an UV detector (\#526, ESA Inc.) at $260 \mathrm{~nm}$ and converted by the CoulArray Analog Input Adapter prior to analysis using the CoulArray ${ }^{\circledR}$ software. All peak areas were within the linear range of the standard curves.

\section{Measurement of Mitochondrial Membrane Potential}

Mitochondrial membrane potential (MMP; $\Delta \psi \mathrm{m}$ ) was determined by confocal microscopy after tetramethylrhodamine ethyl ester (TMRE) staining as previously described (Zhao et al., 2013). After treatment, SH-SY-5Y cells were incubated with TMRE (final concentration: $50 \mathrm{nM}$ ) for $20 \mathrm{~min}$ at $37^{\circ} \mathrm{C}$. The fluorescence was detected using the BD FACS Canto ${ }^{\mathrm{TM}}$ II Flow Cytometry System (BD Biosciences, San Jose, CA, USA). Non-cellular debris and dead cells were gated out, and $\sim 30,000$ events were collected for analysis. Cells were treated with $20 \mu \mathrm{M}$ carbonyl cyanide 4-(trifluoromethoxy) phenylhydrazone (FCCP) for 20 min to collapse $\Delta \psi \mathrm{m}$, and the obtained TMRM fluorescence was used to set the threshold. Data are expressed as the percentage of cells with signal above this threshold.

\section{ROS Assay}

SH-SY-5Y cells at $80 \%$ confluence were treated as above mentioned. Cells were then washed with PBS and incubated with ROS Fluorescent Probe-DHE (Vigorous Biotechnology, Beijing,
China) for $30 \mathrm{~min}$ at $37^{\circ} \mathrm{C}$. After washing thrice with cold PBS, florescence was measured using a microplate reader at the excitation wavelength of $485 \mathrm{~nm}$ and emission wavelength of $525 \mathrm{~nm}$.

\section{Assessment of Mitochondrial Morphology}

SH-SY-5Y cells were grown on poly-D-lysine-coated coverslips. Mitochondria were labeled with DsRed-Mito ( $1 \mu \mathrm{g}$ per $60-\mathrm{mm}$ dish, Clontech, USA) according to manufacturer's instructions. Cells were then fixed with $4 \%$ paraformaldehyde, and coverslips were mounted using Prolong Gold Antifade Reagent with DAPI (Invitrogen). Images were captured using an inverted epifluorescence microscope (Olympus, Tokyo, Japan). Cells with a predominantly intact network of tubular mitochondria were identified as normal ones. Cells with disrupted and predominantly spherical mitochondria were identified as ones with mitochondrial fission. Mitochondrial size and shape were quantified in a blinded manner using ImageJ (NIH image).

\section{Drp1 Translocation}

SH-SY-5Y cells were used to investigate the Drp1 translocation. Cells were cultured on poly-D-lysine-coated coverslips and transfected with $1 \mu \mathrm{g}$ of DsRed-Mito and $1 \mu \mathrm{g}$ of Drp1-myc. After OGD, cells were fixed and immunostained with anti-myc antibody and then with AlexaFluor 488 secondary antibody (Invitrogen). Images were captured and analyzed by confocal microscopy. About 60 cells were analyzed per group in four independent experiments.

\section{Mitochondrial Isolation}

Mitochondria were isolated using the mitochondrial isolation kit (Pierce, Rockford, IL, USA) according to the manufacturer's instructions. Briefly, SH-SY-5Y cells were homogenized in the Dounce homogenizer and then centrifuged at $750 \mathrm{~g}$ for $10 \mathrm{~min}$ at $4^{\circ} \mathrm{C}$. The supernatant was further centrifuged at $12,000 \mathrm{~g}$ for $15 \mathrm{~min}$ at $4^{\circ} \mathrm{C}$, and the pellet was then washed and retained as the mitochondrial fraction.

\section{Enzyme-Linked Immunosorbent Assay (ELISA) Assay}

Brain tissues (the brain regions supplied by MCA) were collected after treatment and rinsed with $1 \times$ PBS. For caspase- 1 activity assay, brain tissues were homogenized in lysis buffer (BioVision, Inc., Milpitas, CA, USA), followed by centrifugation at $10,000 \mathrm{~g}$ for $10 \mathrm{~min}$, and then the supernatant was collected. For in vitro experiments, about $5 \times 10^{6}$ cells were collected, suspended in $50 \mu \mathrm{l}$ of pre-cold lysis buffer, incubated on ice for $10 \mathrm{~min}$, and then centrifuged at $10,000 \mathrm{~g}$ for $1 \mathrm{~min}$, and the supernatant was collected. Protein concentration of the supernatant was determined by Bradford assay. A Caspase-1 Fluorometric Assay kit (BioVision Inc., Milpitas, CA, USA) was used to detect the caspase-1 activity, according to the manufacturers' instructions. The fold change in fluorescence (caspase- 1 activity) as compared to that in the control group was determined.

For the measurement of IL-1 $\beta$, brain tissues (the brain regions supplied by MCA) were homogenized in $20 \mathrm{ml}$ of $1 \times$ PBS, and the lysate was harvested. In addition, cell culture 
media were also collected for the measurement. Samples were centrifuged at $5000 \mathrm{~g}$ for $5 \mathrm{~min}$ and the supernatant was collected. IL- $1 \beta$ concentration was measured using the RayBio ${ }^{\circledR}$ Human IL-1 $\beta$ enzyme-linked immunosorbent assay (ELISA) kit (RayBiotech, Inc., Norcross, GA, USA) according to the manufacturer's instructions. The absorbance at $450 \mathrm{~nm}$ was measured immediately after stop solution was added.

\section{Western Blotting}

The brain regions supplied by MCA were lysed with radioimmunoprecipitation assay buffer (RIPA) containing protease inhibitors (Sigma, St. Louis, MO, USA). The brain tissues were homogenized and sonicated thrice (5 $\mathrm{s}$ for each). All samples were centrifuged at $130,000 \mathrm{~g}$ for $15 \mathrm{~min}$ and the supernatant was collected. Mitochondrial proteins were obtained as described above. Proteins were separated by SDS-PAGE and then transferred to a nitrocellulose membrane which was subsequently blocked in blocking buffer (5\% non-fat milk, $0.1 \%$ BSA, $0.1 \%$ Tween and $34 \mathrm{mmol} / \mathrm{L} \mathrm{NaCl}$, Tris, $\mathrm{pH} 7.5$ ) for $1 \mathrm{~h}$ at room temperature. The membranes were subsequently incubated overnight at $4^{\circ} \mathrm{C}$ with following primary antibodies: anti-Drp1 (1:1000, Cell Signaling Technology, USA); anti-GRP78 (1:1000, Abcam, USA); anti-HSP70 (1:3000, Santa Cruz, USA); anti-HSP60 (1:3000, Santa Cruz); anti-p-PERK (1:2000, Cell Signaling Technology); anti-PERK (1:2000, Cell Signaling Technology); anti-p-eIF2a (1:1000, Cell Signaling Technology); anti-eIF2a (1:1000, Cell Signaling Technology); anti-ATF4 (1:1000, Cell Signaling Technology); anti-CHOP (1:2000, Thermo); anti-caspase12 (1:1000, Cell Signaling Technology); anti-NLRP3 (1:1000, Cell Signaling Technology); anti-TXNIP (1:1000, Abcam); and anti- $\beta$-actin (1:5000, Sigma-Aldrich). Secondary antibodies conjugated with horseradish peroxidase (HRP) were used, and immunoreactivity was visualized by chemiluminescence (SuperSignal Ultra, Pierce, Rockford, IL,
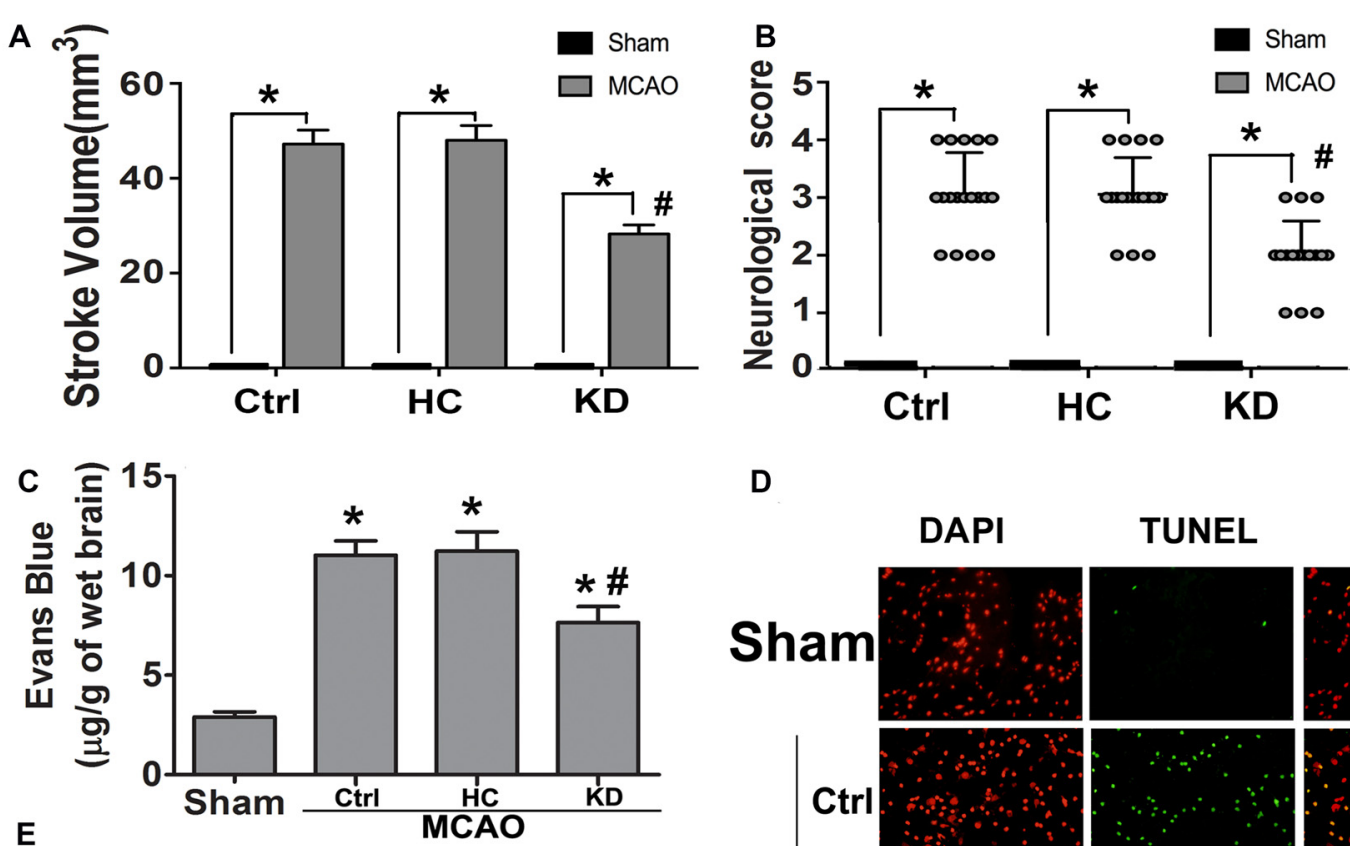

D
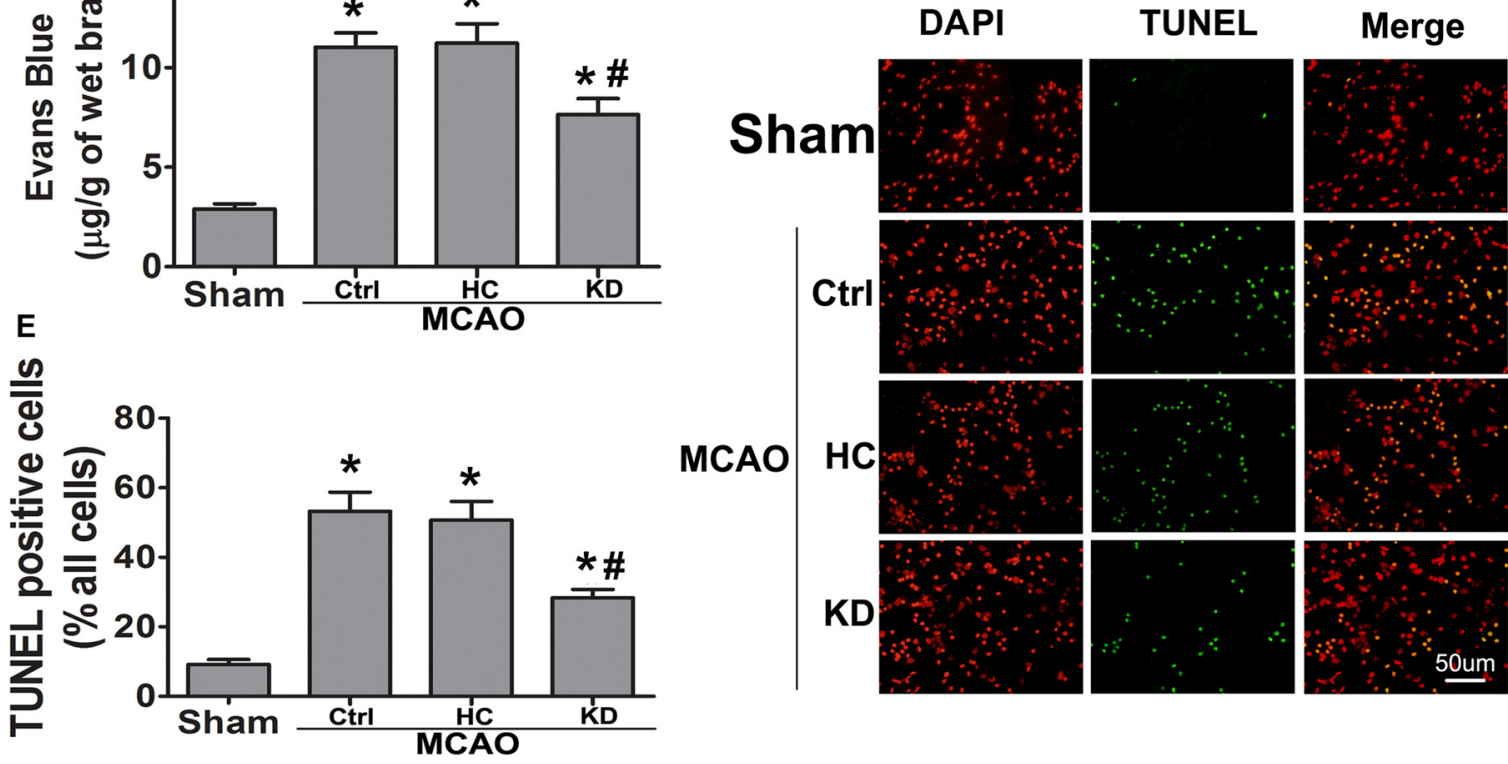

FIGURE 1 | Ketogenic diets (KD) improved ischemic tolerance in middle cerebral artery occlusion (MCAO) mice. Sham-operated and MCAO mice were fed with different diet protocols, and infarct volume (A, $n=6$ per group), neurological score (B, $n=18$ per group), and blood-brain barrier (BBB) permeability (C, $n=6$ per group) were assessed. (D) Fewer TUNEL-positive cells were observed in KD-fed MCAO mice (Scale bar: $50 \mu \mathrm{m}$ ). Brain sections were subjected to TUNEL staining and positive cells were counted (nuclei: DAPI). (E) Quantification of TUNEL-positive cells in different groups ( $n=6$ per group). Data are shown as mean \pm standard error of the mean (SEM); ${ }^{*} P<0.01$ vs. sham-operated mice, ${ }^{\#} P<0.05$ vs. MCAO group. 
USA) assay. Protein bands were analyzed and quantified using Scion Image.

\section{Statistical Analysis}

Data are expressed as mean \pm standard error of the mean (SEM). Comparisons were done with one-way or two-way ANOVA followed by Newman-Keuls post hoc testing for pair-wise comparisons. Statistical analysis was performed using SPSS version 22 (IBM, Armonk, NY, USA). A value of $P<0.05$ was considered statistically significant.

\section{RESULTS}

\section{KD Enhanced Ischemic Tolerance in MCAO Mice}

Following 3-week KD treatment, the daily food intake and calories intake of $\mathrm{KD}$ group gradually decreased while they were gradually increased in HC group Supplementary Figures $\mathrm{S} 1 \mathrm{C}, \mathrm{E})$. However, due to the high-calorie content of $\mathrm{KD}$, there was no difference in total calories intake among the three groups during the 3 weeks (Supplementary Figure S1D). The body weight decreased in the $\mathrm{KD}$ group, but the $\mathrm{BHB}$ level in the KD group was over four times than that in the HC and control groups (Supplementary Figures S1A,B), which was consistent with previously reported ( $\mathrm{Su}$ et al., 2000; Thio et al., 2006). To assess whether KD improved the ischemic tolerance, infarct volume, neurological score, and $\mathrm{BBB}$ permeability were determined in three groups. Results showed KD significantly reduced infarct area and neurological score in MCAO mice (Figures 1A,B). BBB permeability in the $\mathrm{KD}$ group was reduced by $30 \%$ as compared to the control and $\mathrm{HC}$ groups (Figure 1C). Then, the effect of $\mathrm{KD}$ on ischemia-induced cellular apoptosis was further investigated. As shown in Figures 1D,E, fewer TUNEL-positive cells were observed in the KD group as compared to the control or $\mathrm{HC}$ groups. These findings indicate $\mathrm{KD}$ improves ischemic tolerance in MCAO mice.

\section{KD Decreased TXNIP/NLRP3 Inflammasome Induction After Brain Ischemia}

Neuroinflammation plays a significant role in neuronal and glial cell death during ischemic stroke (Seifert and Pennypacker, 2014). The NLRP3 inflammasome is crucial to this inflammatory response, and TXNIP is essential for NLRP3 inflammasome activation. Thus, the TXNIP-NLRP3 pathway was further investigated in MCAO mice. As shown in Figure 2, TXNIP and NLRP3 expression increased in the brain of MCAO mice, which was accompanied by elevated caspase- 1 activity and IL- $1 \beta$ concentration. However, these elevations were inhibited by $\mathrm{KD}$. In mice treated with NLRP3 inflammasome inhibitor CP-456773, NLRP3 inflammasome activation was significantly suppressed, and the caspase-1 activity and IL-1 $\beta$ secretion were also reduced markedly (Figure 3). This protective effect was further investigated in vitro using primary neurons treated with $\mathrm{BHB}$, and results showed BHB suppressed TXNIP/NLRP3 activation
A

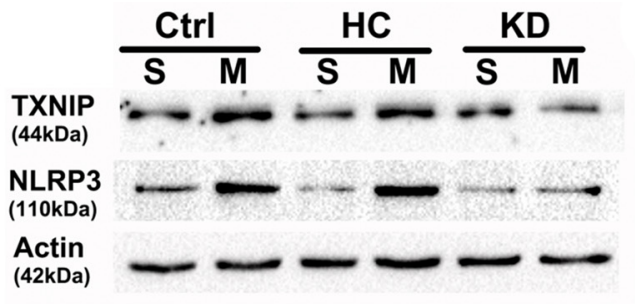

C

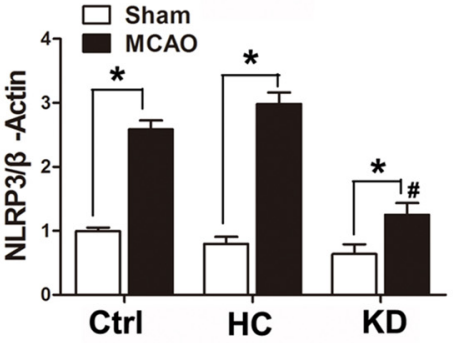

D

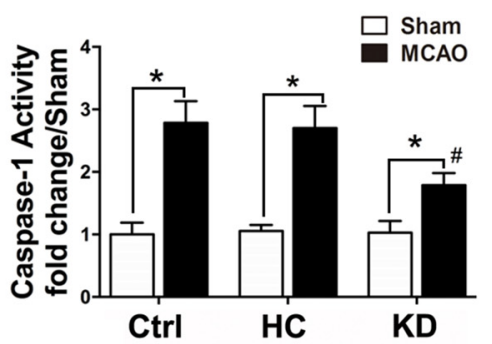

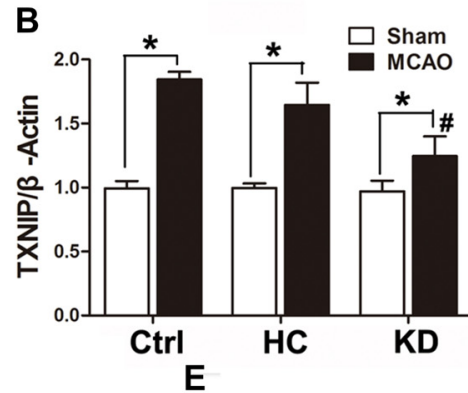

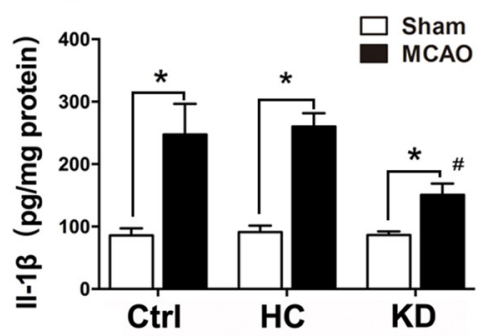

FIGURE 2 | KD suppressed thioredoxin-interacting protein (TXNIP)-nucleotide-binding domain (NOD)-like receptor protein 3 (NLRP3) inflammasome pathway in MCAO mice. Sham-operated and MCAO mice ( $n=6$ per group) were fed with different diet protocols and protein expression was detected by Western blotting. (A) Representative blots from six independent experiments with similar results are shown. TXNIP (B) and NLRP3 (C) protein expression were determined. (D) Caspase-1 activity and IL-1 $\beta$ concentration (E) were determined by enzyme-linked immunosorbent assay (ELISA). Caspase-1 activity was detected on brain lysates and compared to that in sham-operated mice. IL-1 $\beta$ concentration is expressed per mg of total protein in brain tissue lysates. Data are shown as mean \pm SEM; ${ }^{*} P<0.01$ vs. sham-operated mice, ${ }^{\#} P<0.05$ vs. MCAO group. 
A

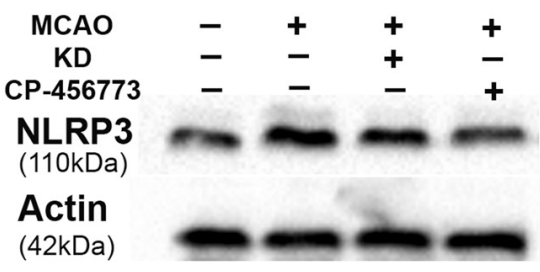

C

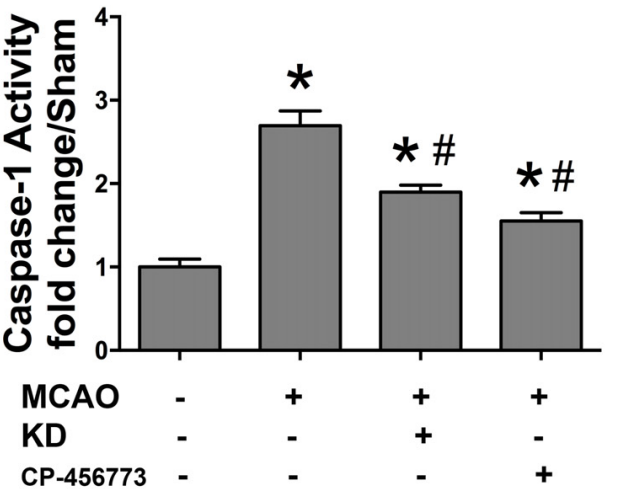

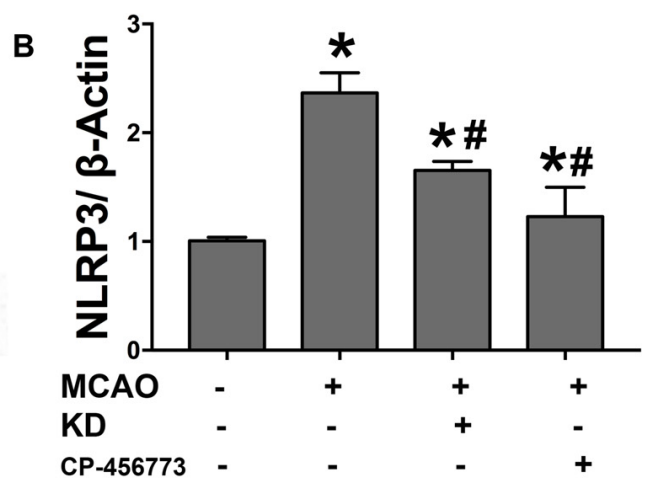

D

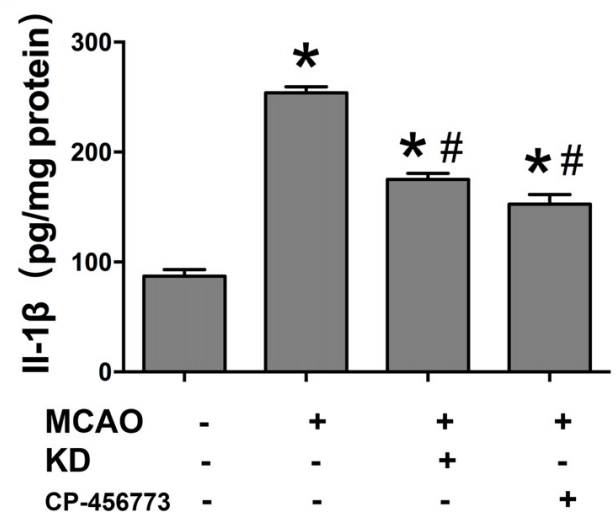

FIGURE 3 | NLRP3 inflammasome inhibitor CP-456773 suppressed NLRP3 mediated inflammation response in MCAO mice. (A,B) Sham-operated and MCAO mice ( $n=6$ per group) were fed with different diet protocols and the protein expression of NLRP3 was detected by Western blotting. Representative blots from six independent experiments with similar results are shown. Caspase-1 activity (C) and IL-1 $\beta$ concentration (D) were detected by ELISA. Caspase-1 activity was determined on brain lysates and compared to that in sham-operated mice. IL-1 $\beta$ production is expressed per mg of total protein in brain tissue lysates. Data are shown as mean \pm SEM; ${ }^{*} P<0.01$ vs. sham-operated mice, ${ }^{\#} P<0.05$ vs. MCAO group.

(Supplementary Figure S2). These findings indicate that KD decreases NLRP3 inflammasome activation after brain ischemia.

\section{BHB Improved Cell Viability and Prevented Mitochondrial Fission in Cells Subjected to OGD/R}

The effects of $\mathrm{BHB}$ on ischemia-related injury were further investigated in an $\mathrm{OGD} / \mathrm{R}$ cell model. $\mathrm{BHB}$ treatment prior to $\mathrm{OGD} / \mathrm{R}$ increased cell viability and decreased $\mathrm{LDH}$ release after OGD/R (Figures 4A,B). Under OGD/R, mitochondrial dynamics towards fission play crucial roles in the NLRP3 inflammasome activation and apoptosis induction (Otera and Mihara, 2012). The balance between mitochondrial fission and fusion controls the mitochondrial morphology and function (Westermann, 2002). Thus, the mitochondrial morphology was investigated in $\mathrm{SH}$ SY-5Y cells. Results showed OGD/R caused significant mitochondrial fragmentation, whereas $\mathrm{BHB}$ prevented the mitochondrial fission (Figure 4C). Moreover, a higher proportion of smaller, rounder mitochondria was present in cells after OGD/R. Consistent with this finding, cells treated with $\mathrm{BHB}$ displayed a higher proportion of tubular, long mitochondria (Figures 4D-F). Because mitochondrial fission was mainly controlled by Drp1, cells were treated with the small molecule mitochondrial division inhibitor (mdivi-1) to inhibit Drp1. These cells showed decreased mitochondrial fragmentation. These findings reveal $\mathrm{OGD} / \mathrm{R}$ likely causes excessive mitochondrial fission, and $\mathrm{BHB}$ may induce the mitochondrial morphological changes by inhibiting Drp1-mediated mitochondrial fission.

\section{BHB Prevented Drp1 Mitochondrial Translocation in Cells Subjected to OGD/R}

SH-SY-5Y cells were used to further investigate the Drp1 mitochondrial translocation. Mitochondria were isolated and Drp1 protein expression was determined. Mitochondrial Drp1 expression increased after $\mathrm{OGD} / \mathrm{R}$, but it was reversed by $\mathrm{BHB}$ pre-treatment (Figures $5 \mathrm{~A}-\mathrm{C}$ ). Drp1 is predominantly cytosolic, and only about $3 \%$ of Drp1 localizes in the mitochondria (Smirnova et al., 2001). Our results indicated Drp1 translocated from the cytosol to the mitochondria after OGD/R. Furthermore, cells were co-transfected with Drp1-myc and DsRed-Mito. OGD/R induced Drp1 aggregation and colocalization 


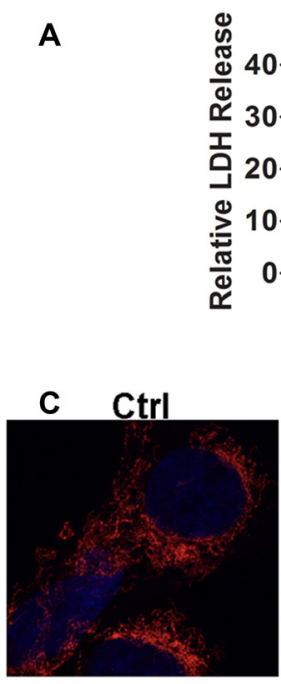

D

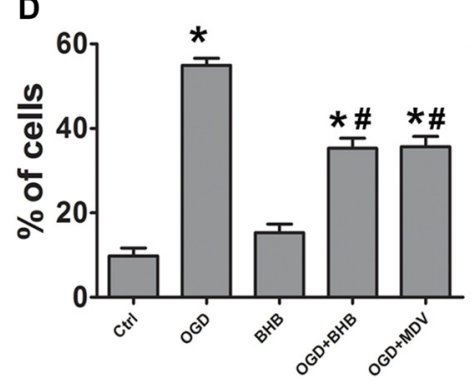

OGD

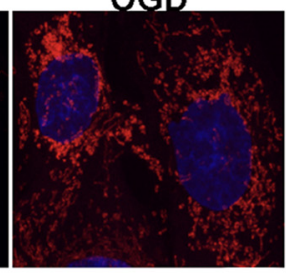

E

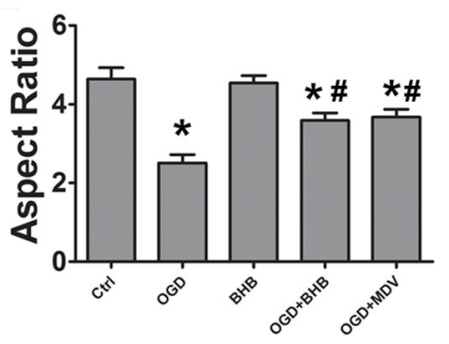

BHB

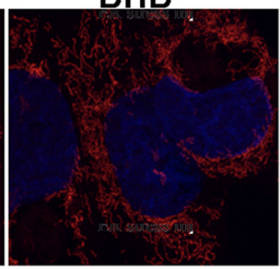

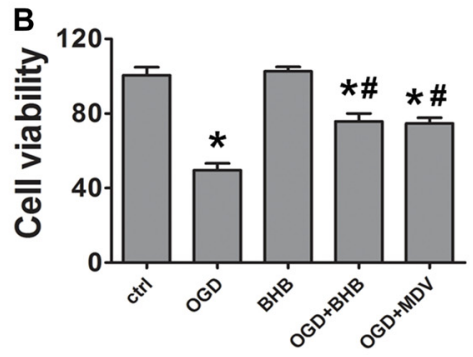

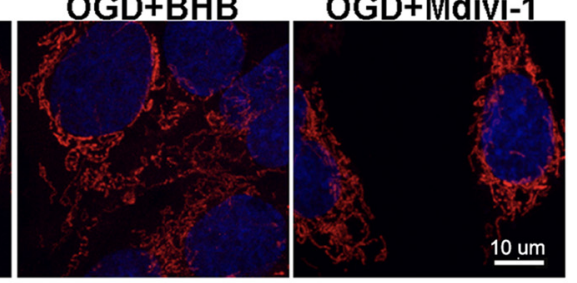

$\mathbf{F}$

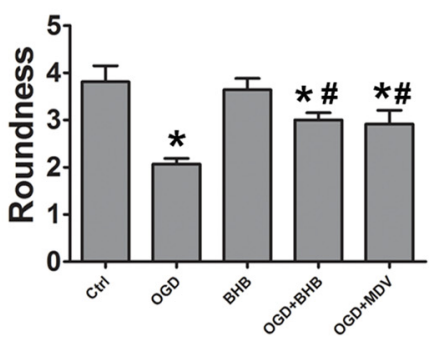

FIGURE 4 | $\beta$-hydroxybutyrate (BHB) and mdivi-1 influenced cell survival and mitochondrial morphology in response to oxygen-glucose deprivation/reoxygenation (OGD/R) damage. BHB and mdivi-1 treatment inhibited lactate dehydrogenase (LDH) release (A) and improved cell viability (B) after OGD/R. Data were from six independent experiments. (C) Cultured SH-SY-5Y cells were labeled with MitoDs-red for the evaluation of mitochondrial morphology. Scale bar: $10 \mu m$ (D) Percentage of cells with truncated or fragmented mitochondria. Quantification was performed in a blind manner from more than 200 cells in at least 10 randomly selected fields at $200 \times$ from three independent experiments. (E,F) Quantification of mitochondrial morphology. Roundness was calculated as perimeter ${ }^{2} / 4 \pi$ area. Aspect ratio is a measurement of major/minor axes. Both of these values approach 1 as the particle becomes more circular. Quantification was performed from 10 to 15 randomly selected cells in three independent experiments. Data are shown as mean $\pm \mathrm{SEM}$; $P<0.01$ vs. Ctrl group, ${ }^{*} P<0.05$ vs. OGD group.

with the mitochondria, but BHB significantly reduced Drp1 mitochondrial translocation, similar to the findings after mdivi-1 pre-treatment (Figures 5D,E).

\section{BHB Improved Mitochondrial Function and Suppressed NLRP3 Inflammasome Activation by Reducing ROS Production in Cells Subjected to OGD/R}

To determine whether the maintenance of mitochondrial function is one of the mechanisms by which $\mathrm{BHB}$ exerts a protective effect on OGD/R, the ATP level in SH-SY-5Y cells was detected by HPLC, and a fluorescent cationic dye was used to measure the $\Delta \psi \mathrm{m}$. Oligomycin, an inhibitor of the electron transport chain complex $\mathrm{V}$, was used as a positive control for ATP measurement. As expected, OGD/R significantly reduced the ATP level, which, however, was partially reversed by $\mathrm{BHB}$ pre-treatment (Figure 6A). Moreover, BHB conferred protection against $\Delta \psi \mathrm{m}$ reduction induced by $\mathrm{OGD} / \mathrm{R}$ (Figure 6B).
Because excessive ROS are generated in dysfunctional mitochondria, the effect of $\mathrm{BHB}$ on ROS production was further investigated. BHB pre-treatment decreased ROS production (Figure 6C). ROS is a strong NLRP3 inflammasome activator. Our results showed $\mathrm{BHB}$ and ROS inhibitor NAC (NAC) pre-treatment attenuated NLRP3 expression as well as reduced caspase- 1 activity and IL- $1 \beta$ release (Figures $6 \mathrm{D}-\mathrm{G}$ ) after OGD/R. These results indicate that $\mathrm{BHB}$ suppresses NLRP3 activation by inhibiting ROS production in cells subjected to OGD/R.

\section{KD Attenuated ER Stress After Brain Ischemia}

Ischemia and hypoxia commonly cause ER stress due to the reduced protein folding capacity of ER, thus leading to the accumulation of misfolded proteins. ER stress involves three main signaling pathways: PERKeIF2-ATF4, IRE1-XBP1 and ATF6 pathways (Xin et al., 2014). The protein expression of p-PERK, p-eIF2 $\alpha$ and 

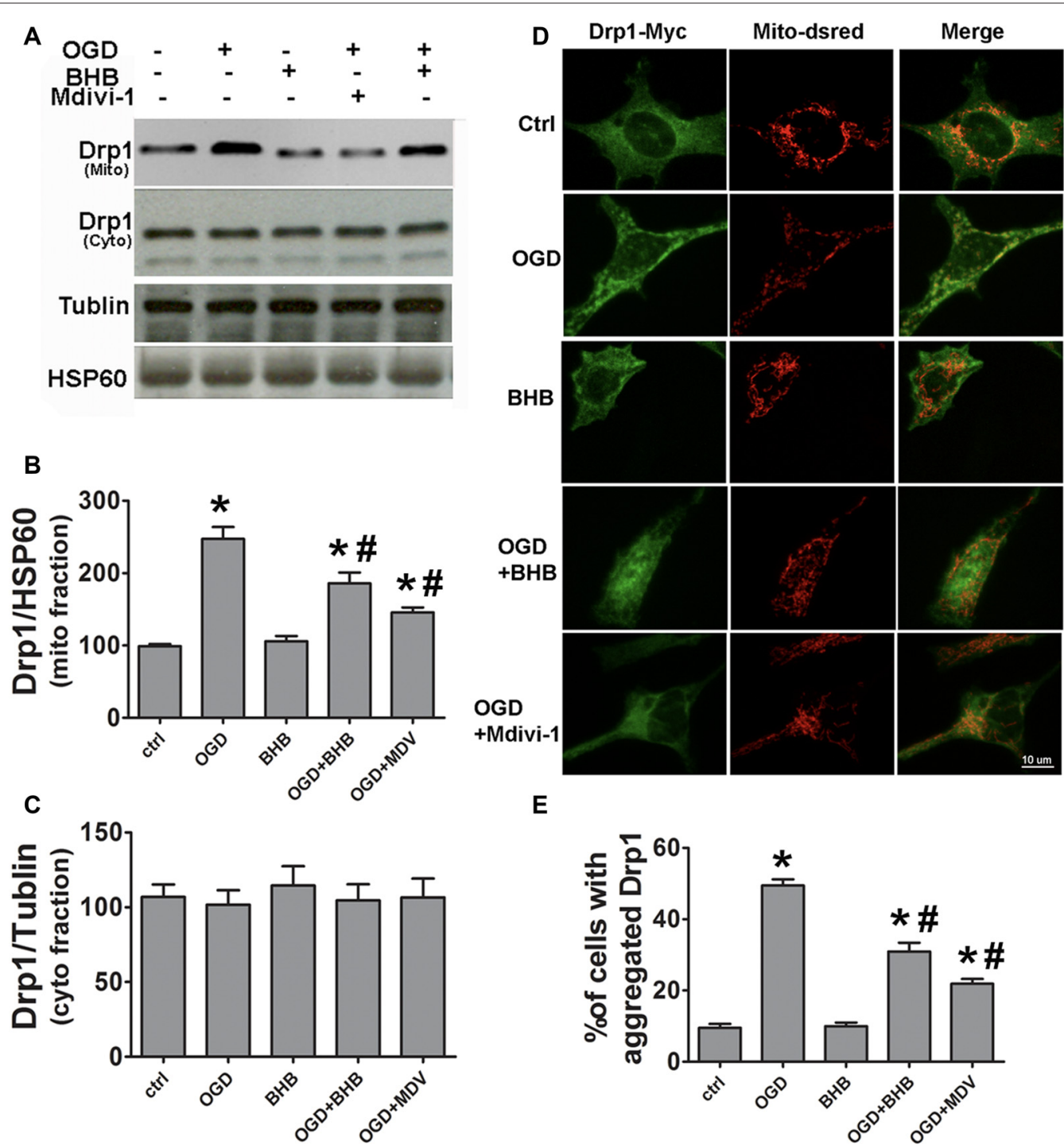

E

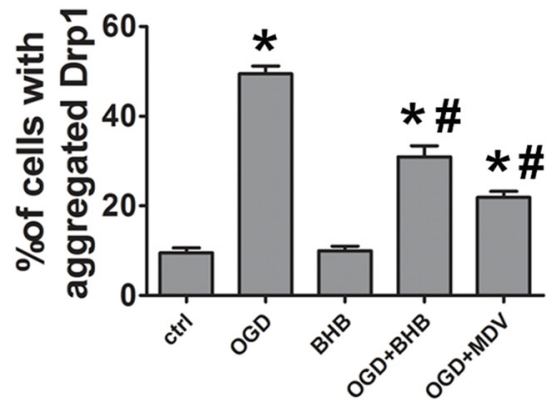

FIGURE 5 | BHB and mdivi-1 prevented dynamin-related protein 1 (Drp1) mitochondrial translocation after OGD/R. (A-C) Protein expression of mitochondrial fission protein Drp1 in the mitochondria and cytoplasm was detected by Western blotting. Representative blots from six independent experiments with similar results are shown. (D) SH-SY-5Y cells were co-transfected with DsRed-Mito and Drp1-myc. Scale bar: $10 \mu \mathrm{m}$. (E) Quantification of aggregated Drp1 in cells subjected to different treatments. Data are shown as mean $\pm \mathrm{SEM}$; ${ }^{*} P<0.01$ vs. Ctrl group, ${ }^{\#} P<0.05$ vs. OGD group.

ATF4 was detected by Western blotting in this study. Results showed ischemia significantly increased the protein expression of p-PERK, p-eIF2 $\alpha$ and ATF4 in the brain of MCAO mice. However, the activation of p-PERK-p-eIF2 $\alpha$-ATF4 pathway was inhibited in the $\mathrm{KD}$ group (Figure 7).

GRP78, a chaperone protein in ER stress, can inhibit protein misfolding and exert negative regulatory effects on ER stress. HSP70 is an important chaperone regulated by ER stress. In this study, the GRP78 and HSP70 expression was higher in the $\mathrm{KD}$ group (Figures $\mathbf{8 A}-\mathbf{C}$ ) than in other two groups. CHOP and cleaved caspase-12 are ER-specific apoptotic promoters. The expression of both $\mathrm{CHOP}$ and cleaved caspase-12 markedly reduced, and pro-caspase-12 protein expression increased in KD-treated mice with MCAO (Figures 8D-G). These findings indicate that $\mathrm{ER}$ stress is attenuated in $\mathrm{KD}$-treated mice with MCAO.

\section{BHB Suppressed NLRP3 Inflammasome Activation by Down-Regulating ER Stress in Cells Subjected to OGD/R}

Because ER stress can activate the NLRP3 inflammasome (Menu et al., 2012), the NLRP3 expression, IL- $1 \beta$ content and caspase- 1 activity were further detected in SH-SY-5Y cells subjected to OGD/R. Our results showed OGD triggered NLRP3 activation, as observed after the use of ER stress agonist tunicamycin. However, 


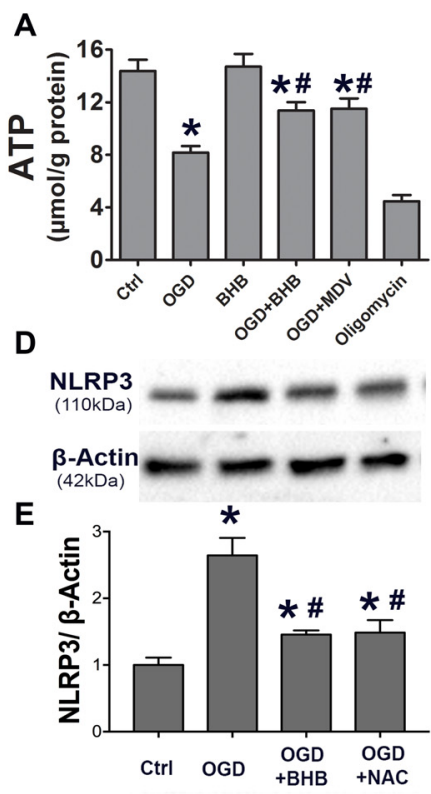

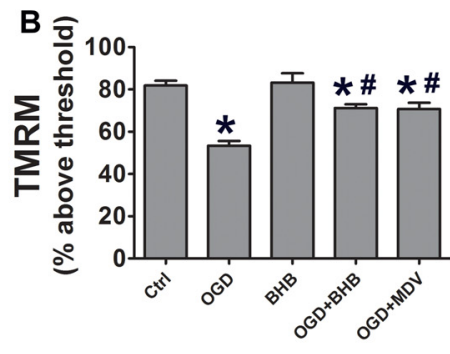

$\mathbf{F}$

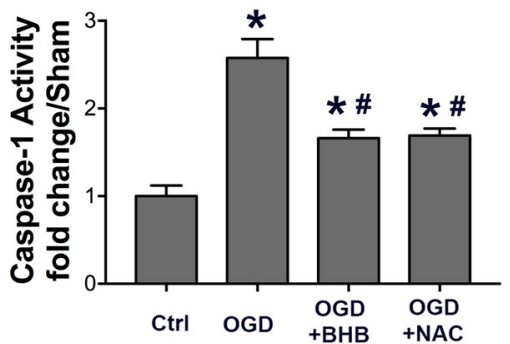

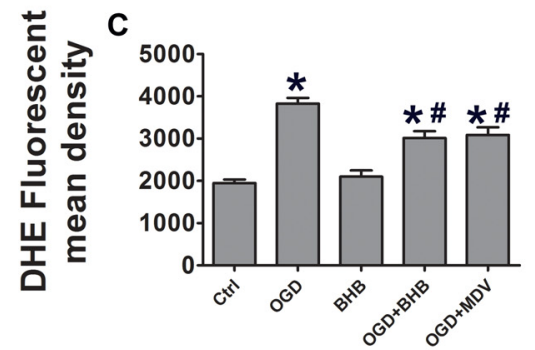

G

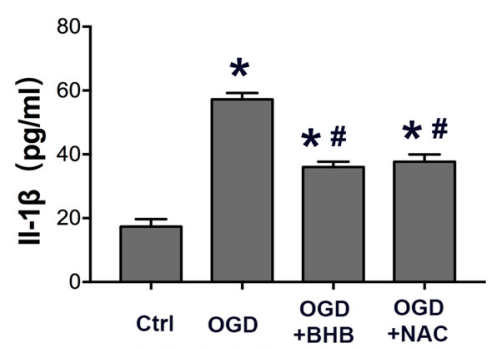

FIGURE 6 | BHB and mdivi-1 prevented mitochondrial function and reactive oxygen species (ROS) inhibitor N-acetyl-L-cysteine (NAC) suppressed NLRP3 inflammasome after OGD/R. (A) BHB and mdivi-1 reversed the decreased ATP production after OGD/R. Oligomycin (oligo, $10 \mu \mathrm{M}$, a complex $V$ inhibitor), as a positive control, significantly reduced ATP level. Data were normalized to those from oligomycin-treated cells. BHB and mdivi-1 reversed the increase in ROS production (C) and the decrease in mitochondrial membrane potential (MMP) (B) after OGD/R. (D,E) The ROS inhibitor NAC was added before OGD, and the protein expression of NLRP3 was detected by Western blotting. Representative blots from six independent experiments with similar results are shown. (F) Caspase-1 activity was detected on cell lysates and compared to that in sham-operated mice. (G) IL-1 $\beta$ production in culture media. Data are shown as mean \pm SEM; ${ }^{*} P<0.01$ vs. Ctrl group, ${ }^{\#} P<0.05$ vs. OGD group.
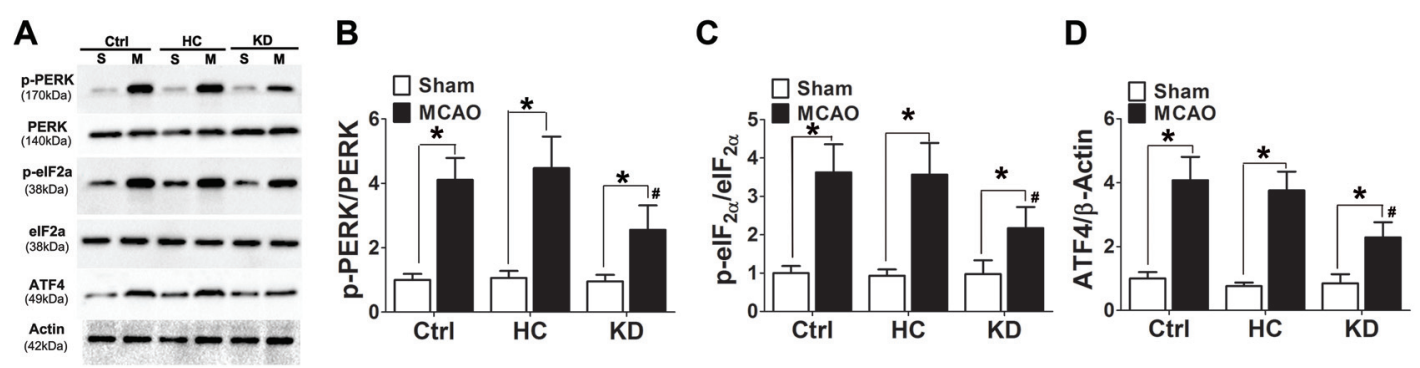

FIGURE 7 | KD suppressed the p-PERK-p-elF2 $\alpha$-ATF4 pathway in MCAO mice. Sham-operated and MCAO mice ( $n=6$ per group) were fed with different diet protocols and Western blotting (A) was used to determine the protein expression of p-PERK/PERK (B), p-elF2a/elF2a (C) and ATF4/ $\beta$-actin (D). Representative blots from six independent experiments with similar results are shown. Data are shown as mean $\pm \mathrm{SEM}$; ${ }^{*} P<0.01$ vs. sham-operated mice, ${ }^{\#} P<0.05$ vs. MCAO group.

BHB pre-treatment significantly inhibited NLRP3 expression, IL-1 $\beta$ release, and caspase-1 activity. Further, the ER stress TUDCA also suppressed NLRP3 activation in vitro (Figure 9), indicating that $\mathrm{BHB}$ is able to suppress NLRP3 inflammasome activation.

\section{DISCUSSION}

Inflammation-mediated cell dysfunction is an initial event in the ischemic stroke, and contributes to the development of neurological deficits (Tobin et al., 2014). The results of the present study showed that, under ischemic conditions, cellular
NLRP3 inflammasome was activated by hypoxia-ischemia insult, thus leading to brain tissue damage. Moreover, KD pre-treatment suppressed NLRP3 inflammasome activation by inhibiting Drp1-mediated mitochondrial fission and ER stress, which alleviated brain dysfunction in ischemic stroke.

$\mathrm{KD}$ is a high-fat diet in which carbohydrates are nearly eliminated, and it provides sufficient protein for growth but insufficient amounts of carbohydrates for the body's metabolic needs. Thus, the oxidation of a large amount of fat in liver mitochondria leads to elevated circulating KBs (acetoacetate, $\beta$-hydroxybutyrate and acetone; 

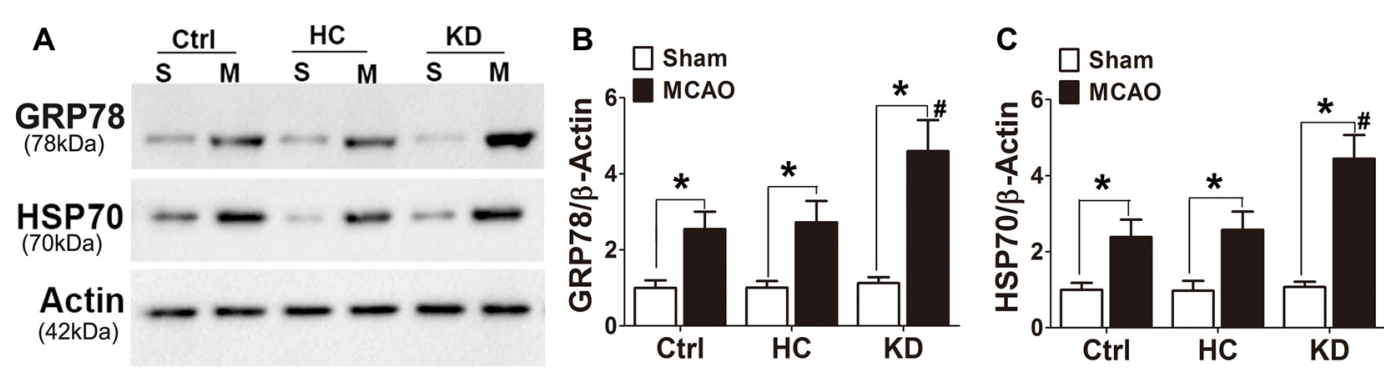

D

E $\mathrm{Sham}$

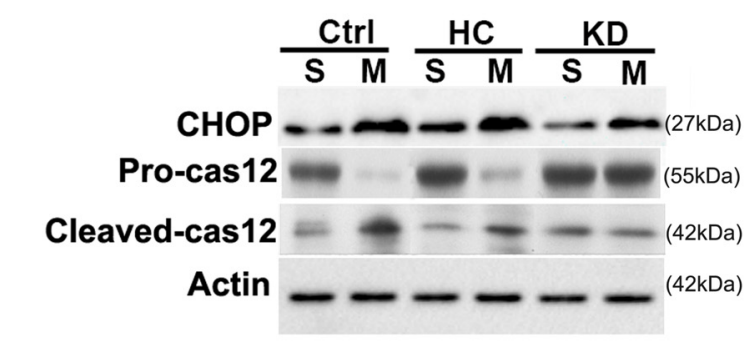

F

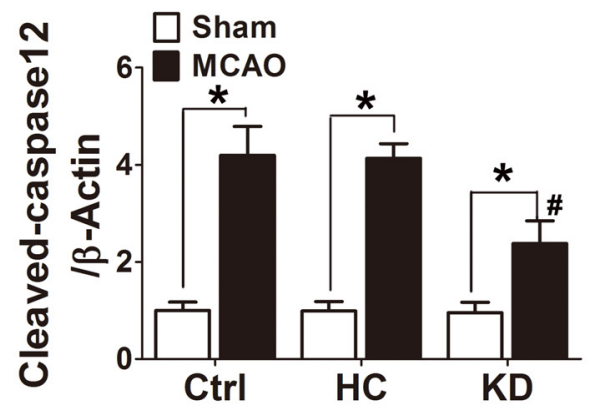

G

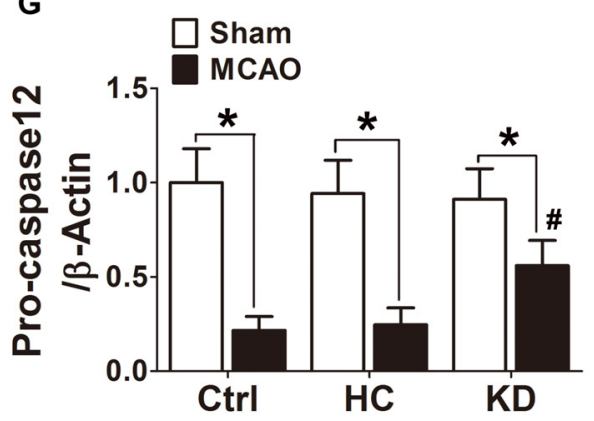

FIGURE 8 | KD increased GRP78/HSP70 level but attenuated CHOP and cleaved caspase 12 expression in MCAO mice. Sham-operated and MCAO mice ( $n=6$ in each group) were fed with different diet protocols and Western blotting (A,D) was performed to determine the protein expression of GRP78 (B), HSP70 (C), CHOP (E), cleaved-caspase $12(\mathbf{F})$ and pro-caspase $12(\mathbf{G})$. Representative blots from six independent experiments with similar results are shown. Data are shown as mean \pm SEM; ${ }^{*} P<0.01$ vs. sham-operated mice, ${ }^{\#} P<0.05$ vs. MCAO group.

Gasior et al., 2006). Eventually, KBs in the circulation readily cross the $\mathrm{BBB}$ and are consumed as an alternative fuel in the brain under some conditions such as fasting, extensive physical exercise and $\mathrm{KD}$ (Hartman et al., 2007). In fact, the capability of brain to consume KBs is considered as a form of cerebral metabolic adaptation (Prins, 2008).

$\mathrm{KD}$, which has been successfully used to treat drug-resistant epilepsy, has been found to confer neuroprotective effects on the cerebral ischemic injuries (Shaafi et al., 2014). Actually, in animal studies, a ketogenic state (induced by $\mathrm{KD}$, exogenously administered $\mathrm{KBs}$ or calorie restriction) is proven to exert an overall significant protective effect on outcomes (decreased lesion volume, neurological score and edema, and improved survival, neuronal count and behavior) following brain ischemia in various models, including the MCAO model, vessel occlusion model and cardia arrest model (Gibson et al., 2012). Both chronic interventions like calorie restriction for 3 months or KD for 3 weeks and acute interventions like calorie restriction for
4 days or ketone administration from several days to $30 \mathrm{~min}$ before the onset of experimental stroke can exert protective effects (Suzuki et al., 2001; Puchowicz et al., 2008; Tai et al., 2008; Yoon et al., 2011). Moreover, it is also beneficial for the adoption to calorie restriction $1 \mathrm{~h}$ after ischemia and supplement with exogenous ketones immediately or $30 \mathrm{~min}$ after ischemic event (Suzuki et al., 2002; McEwen and Paterson, 2010). In the present study, our results also indicate that 3-week KD was able to enhance the brain ischemic tolerance to MCAO in mice, as demonstrated by improvements of infarct area, neurological score, BBB permeability and cellular apoptosis.

In spite of emerging evidence from preclinical investigations demonstrating the beneficial effects of $\mathrm{KD}$, mechanisms underlying its effects are not well studied. Limited evidence from precious studies suggest that $\mathrm{KD}$ is able to alleviate excitotocity, oxidative stress and apoptosis (Shaafi et al., 2014). Puchowicz et al. (2008) found the hypoxia inducible factor $1 \alpha(\mathrm{HIF}-1 \alpha)$ level was elevated after 3-week KD 
A

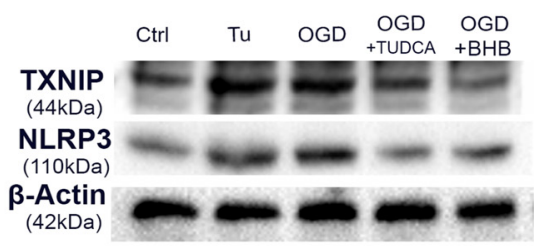

C

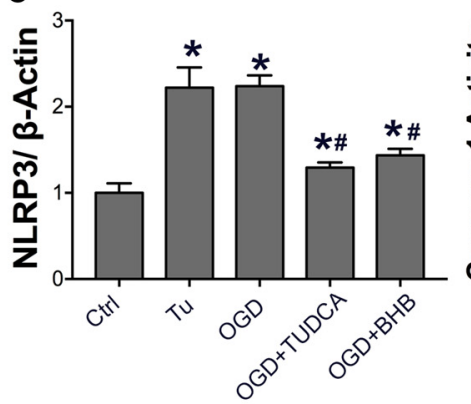

D

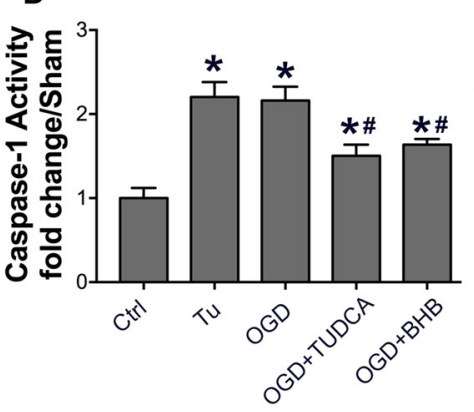

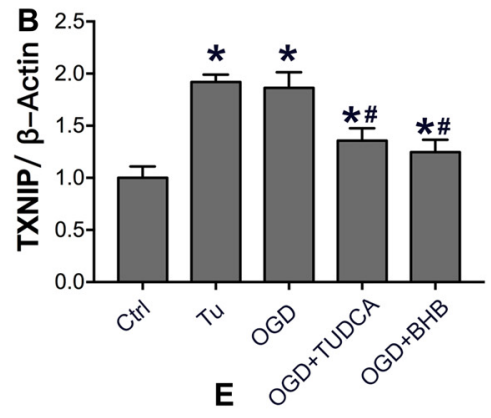

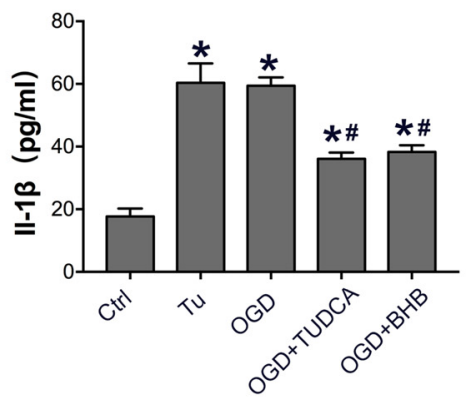

FIGURE 9 | BHB and endoplasmic reticulum (ER) stress inhibitor tauroursodeoxycholic acid (TUDCA) suppressed NLRP3 inflammasome after OGD/R. (A-C) Protein expression of TXNIP and NLRP3 was detected by Western blotting. Representative blots from six independent experiments with similar results are shown.

(D) Caspase-1 activity was detected on cell lysates and compared to that in sham-operated mice. (E) IL-1 $\beta$ concentration of culture medium. Data are shown as mean \pm SEM; ${ }^{*} P<0.01$ vs. Ctrl group, ${ }^{\#} P<0.05$ vs. OGD group. Tu, tunicamycin.

treatment because increased succinate inhibited the prolylhydroxylase, an enzyme responsible for the degradation of HIF. Our previous study reported the transcription of HIF target genes including erythropoietin (EPO), endothelial cell growth factor (VEGF), Glut-1 and MCT4 increased and this increase was partly related to the elevated HIF expression (Yang et al., 2017). Moreover, Ly-6CLo monocytes and/or macrophages express hydroxy-carboxylic acid receptor 2 (HCA2), a receptor for $\mathrm{BHB}$, which has been reported to reduce neuroinflammation and exert a protective effect on the brain injury after KD (Rahman et al., 2014; Offermanns and Schwaninger, 2015). Other inflammatory responses, especially NLRP3 inflammasome, are also important in post-ischemic damage. Nevertheless, the effects of $\mathrm{KD}$ on NLRP3 inflammasome in the central nerves system remain unclear.

The NLRP3 inflammasome is an important component in innate immune response, and contributes significantly to ischemic brain injury following stroke. The NLRP3 inflammasome is activated following cerebral ischemia, and pharmacological inhibition of NLRP3-mediated inflammatory response can prevent the deterioration of cerebral function (Fann et al., 2013a,b). In this study, whether the neuroprotective effects of $\mathrm{KD}$ pre-treatment were related to the inhibition of NLRP3-mediated inflammatory response was further investigated. Our results revealed that MCAO and OGD induced TXNIP/NLRP3 inflammasome activation and significantly increased caspase- 1 activity and IL-1 $\beta$ release. However, $\mathrm{KD}$ or $\mathrm{BHB}$ pre-treatment significantly suppressed the NLRP3-mediated inflammatory response, as confirmed after use of the NLRP3 inhibitor CP456773. However, the specific mechanism by which KD regulates NLRP3 inflammasome remains still unclear.

Although increasing evidence suggests that mitochondria serve as a regulator of NLRP3 inflammasome activation by producing mitochondrial ROS or DNA in response to damage (Zhou et al., 2011), the role of mitochondrial dynamics in the inflammasome pathway in neurons is still unknown. Our results indicated that Drp1 acted a downstream factor regulated by KD and could regulate the NLRP3 inflammasome activation during ischemic injury. Drp1, normally found in the cytosol, is a GTPase that mediates mitochondrial fission (Archer, 2013). Phosphorylated Drp1 can translocate to the mitochondrial outer membrane, where it interacts with the mitochondrial fission protein Fis1 to induce mitochondrial fission, thus generating ROS and activating NLRP3 inflammasome (Knott et al., 2008; Archer, 2013). Our results showed OGD/R induced Drp1 mitochondrial translocation to cause aberrant mitochondrial fission, thus leading to the significant NLRP3 inflammasome activation. In contrast, BHB pre-treatment suppressed Drp1 translocation and mitochondrial fragmentation, attenuating NLRP3 inflammasome activation. These results were consistent with previously reported that $\mathrm{BHB}$ could block the NLRP3 inflammasome activation (Youm et al., 2015). In addition, abnormal mitochondrial function, including decreased ATP generation and MMP, could be reversed by $\mathrm{BHB}$ as well as Drp1 inhibitor mdivi-1. Taken together, our results provide a molecular insight into the effects of $\mathrm{BHB}$ on 
the mitochondrial dynamics-regulated NLRP3 inflammasome activation.

It has been proposed that increased ROS due to mitochondrial dysfunction can be sensed by the complex TXNIP and then induce the dissociation of this complex (Gross et al., 2011). In normal cells, TXNIP is constitutively maintained in the reduced state; in case of increased ROS production, this complex dissociates and TXNIP binds to the LRR region of NLRP3, leading to the NLRP3 inflammasome activation (Tschopp and Schroder, 2010). In the present study, BHB pre-treatment reduced ROS generation in cells subjected to $\mathrm{OGD} / \mathrm{R}$, and $\mathrm{BHB}$ or ROS inhibitor NAC could attenuate the activation of TXNIP/NLRP3 inflammasome, indicating that BHB may protect cells against ROS-mediated NLRP3 activation following OGD/R.

ER stress is an essential progress in the brain ischemiareperfusion injury (Roussel et al., 2013). The ER stress sensors PERK and IRE1 $\alpha$ can induce TXNIP to activate the NLRP3 inflammasome (Lerner et al., 2012; Oslowski et al., 2012). IRE1 $\alpha$ also induces ROS-dependent NLRP3 translocation to mitochondria, leading to the release of mitochondrial signals that further activate the inflammasome (Bronner et al., 2015). As expected, the p-PERK-p-eIF2 $\alpha$-ATF4 pathway was activated and $\mathrm{CHOP}$ expression increased significantly in the brain of MCAO mice, suggesting ER stress, which was significantly attenuated in the $\mathrm{KD}$ group. In vivo, the $\mathrm{ER}$ stress inducer tunicamycin or OGD/R activated the NLRP3 inflammasome, but $\mathrm{BHB}$ pre-treatment reduced this inflammation response, which was similar to the effects of ER stress inhibitor TUDCA. Collectively, these results indicate that $\mathrm{KD}$ or $\mathrm{BHB}$ is able to ameliorate ER stress-associated NLRP3 inflammasome activation.

When considering $\mathrm{KD}$ as a preventive therapy for high risk population of stroke, we must take into account the clinical safety, especially if the high cholesterol content of KD may cause dyslipidemia and increase the risk for stroke. It was reported that the postprandial triacylglycerol (TG) reduced, oxidized low density lipoprotein (LDL) remained unchanged and the high-density lipoprotein increased after 6-week KD treatment in normal weight men (Sharman et al.,

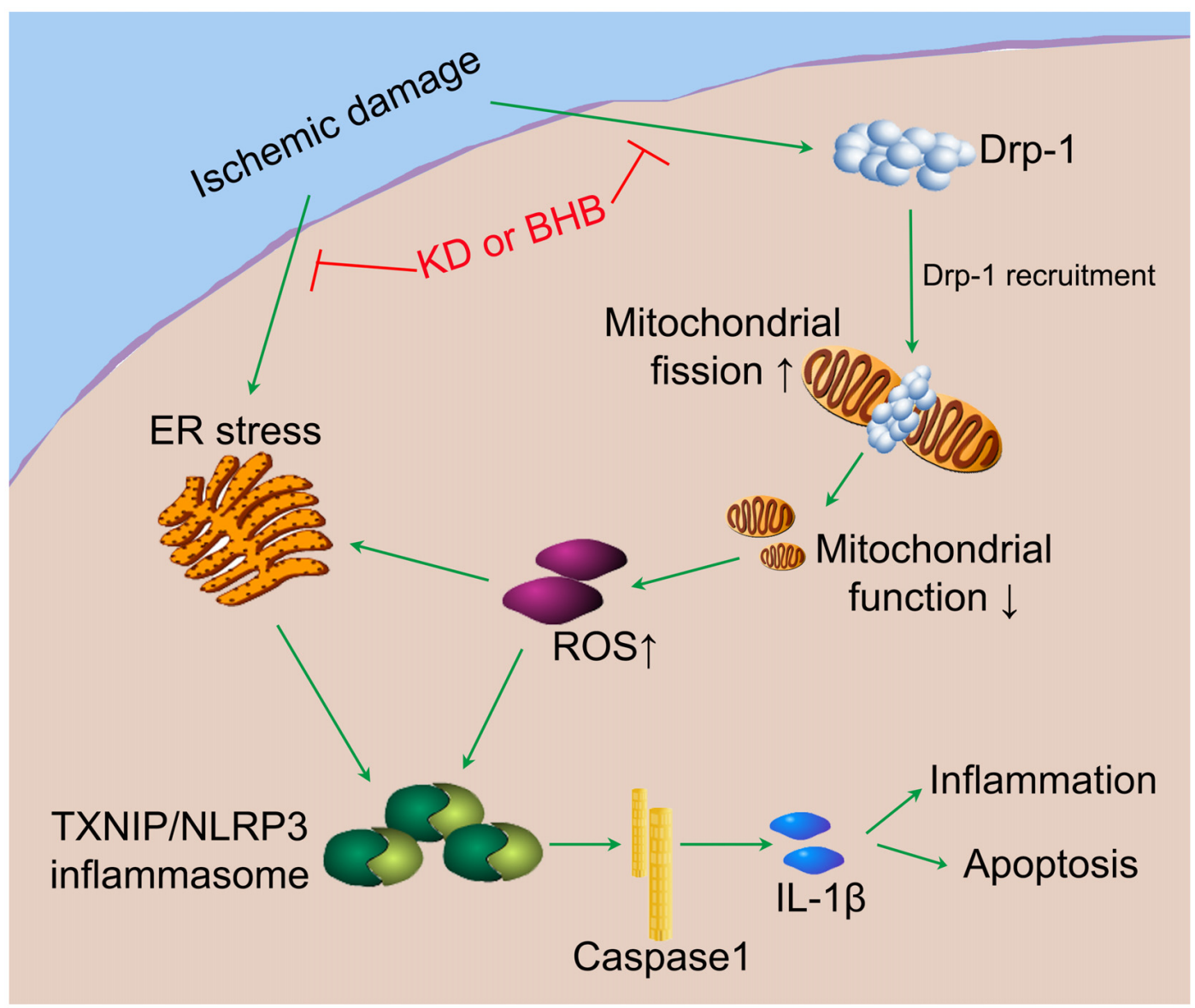

FIGURE 10 | The potential mechanistic pathway by which KD or BHB regulates NLRP3 inflammasome activation. Ischemic damage causes Drp1-mediated mitochondrial fission and ER stress. Excessive ROS production aggravates ER stress and induces TXNIP/NLRP3 activation. The resultant maturation of IL-1 $\beta$ is responsible for the inflammation and cell apoptosis, leading to the central nervous system dysfunction. KD or BHB suppresses Drp1 recruitment and ER stress, thereby alleviating inflammation and improving brain ischemic injury. 
2002). Furthermore, KD shifts the LDL particle distribution to a larger size, resulting in significant increases in peak and mean LDL diameter and decrease in the proportion of small, dense LDL particles (Meckling et al., 2002; Sharman et al., 2002; Dashti et al., 2003; Volek et al., 2005), which is considered beneficial because small LDL particles are atherogenic. In fact, the majority of recent studies demonstrate that physiological ketosis can actually benefit the blood lipid profiles (Brehm et al., 2003; Shai et al., 2008; Volek et al., 2009).

There were several limitations in this study. First, in the in vitro study, $\mathrm{BHB}$ was used to mimic the $\mathrm{KD}$ in the in vivo study. $\mathrm{KD}$ was adopted in animals in order to achieve a prolonged neuroprotective state before ischemia. Although $\mathrm{BHB}$ is the major component of $\mathrm{KBs}$ and the most widely used ingredient in vitro, the possibility that the mice are also protected by components other than BHB can not be excluded, because KD has multiple ingredients. Second, we did not assess the effects of other KBs like acetoacetate in vitro, thus whether other KBs work in the same way as BHB remains still unclear. Further studies are needed to elucidate these issues.

\section{CONCLUSION}

Our results indicate that $\mathrm{KD}$ or $\mathrm{BHB}$ can prevent NLRP3 inflammasome activation and thereby improve the brain ischemic tolerance. As summarized in Figure 10, KD or BHB may inhibit Drp1 mitochondrial translocation to protect mitochondrial morphology and function, which prevents ROS-associated NLRP3 inflammasome activation. Further, BHB treatment may also ameliorate ER stressassociated NLRP3 inflammasome activation. Although prophylactic KD therapy is still not used in clinical settings, our findings confirm the neuroprotective effects of $\mathrm{KD}$ and provide evidence on the potential molecular mechanism underlying these effects, which support the potential clinical

\section{REFERENCES}

Archer, S. L. (2013). Mitochondrial dynamics-mitochondrial fission and fusion in human diseases. N. Engl. J. Med. 369, 2236-2251. doi: 10.1056/NEJMra1215233

Bae, H. R., Kim, D. H., Park, M. H., Lee, B., Kim, M. J., Lee, E. K., et al. (2016). $\beta$-Hydroxybutyrate suppresses inflammasome formation by ameliorating endoplasmic reticulum stress via AMPK activation. Oncotarget 7, 66444-66454. doi: 10.18632/oncotarget.12119

Barrett, K. M., Lal, B. K., and Meschia, J. F. (2015). Stroke: advances in medical therapy and acute stroke intervention. Curr. Cardiol. Rep. 17:79. doi: 10.1007/s11886-015-0637-1

Bertholet, A. M., Delerue, T., Millet, A. M., Moulis, M. F., David, C., Daloyau, M., et al. (2016). Mitochondrial fusion/fission dynamics in neurodegeneration and neuronal plasticity. Neurobiol. Dis. 90, 3-19. doi: 10.1016/j.nbd.2015. 10.011

Brehm, B. J., Seeley, R. J., Daniels, S. R., and D'Alessio, D. A. (2003). A randomized trial comparing a very low carbohydrate diet and a calorierestricted low fat diet on body weight and cardiovascular risk factors in healthy women. J. Clin. Endocrinol. Metab. 88, 1617-1623. doi: 10.1210/jc.2002021480 utility of $\mathrm{KD}$ as nutritional supply in acute cerebrovascular diseases.

\section{AVAILABILITY OF DATA AND MATERIALS}

All data in this study are included in this published article and its supplementary documents.

\section{AUTHOR CONTRIBUTIONS}

MG and XW: contributed equally to this work. XC and $\mathrm{MC}$ : co-correspondence for this article. MC, YZ and QD: conceived and designed the experiments. MG, XW and QY: performed the experiments. MG, QD and HD: analyzed the data. XC, QY, HD and YZ: contributed reagents/materials/analysis tools. MG and MC: drafted the article.

\section{FUNDING}

This study was supported by the National Natural Science Foundation of China (81471173 to MC; 81571109 to QD; 81571033 to YZ), Grant from the Science and Technology Commission of Shanghai Municipality (16411970200), Key Basic Research Grant from the Science and Technology Commission of Shanghai Municipality (16JC1400500), International Science and Technology Cooperation Program of China (2014DFA32830), Key Research and Development Plants of Jiangsu Province (BE2016726) and the National Key Research and Development Program of China (2017YFC0907002, 2017YFC0907501)

\section{SUPPLEMENTARY MATERIAL}

The Supplementary Material for this article can be found online at: https://www.frontiersin.org/articles/10.3389/fnmol.2018. 00086/full\#supplementary-material

Bronner, D. N., Abuaita, B. H., Chen, X., Fitzgerald, K. A., Nuñez, G., He, Y., et al. (2015). Endoplasmic reticulum stress activates the inflammasome via NLRP3- and caspase-2-driven mitochondrial damage. Immunity 43, 451-462. doi: 10.1016/j.immuni.2015.08.008

Camberos-Luna, L., Gerónimo-Olvera, C., Montiel, T., Rincon-Heredia, R., and Massieu, L. (2016). The ketone body, $\beta$-hydroxybutyrate stimulates the autophagic flux and prevents neuronal death induced by glucose deprivation in cortical cultured neurons. Neurochem. Res. 41, 600-609. doi: 10.1007/s11064015-1700-4

Cui, M., Tang, X. N., Christian, W. V., Yoon, Y., and Tieu, K. (2010). Perturbations in mitochondrial dynamics induced by human mutant PINK1 can be rescued by the mitochondrial division inhibitor mdivi-1. J. Biol. Chem. 285, 11740-11752. doi: 10.1074/jbc.M109.066662

Dashti, H. M., Bo-Abbas, Y. Y., Asfar, S. K., Mathew, T. C., Hussein, T., Behbahani, A., et al. (2003). Ketogenic diet modifies the risk factors of heart disease in obese patients. Nutrition 19, 901-902. doi: 10.1016/s08999007(03)00161-8

Doerfler, A., Schwab, S., Hoffmann, T. T., Engelhorn, T., and Forsting, M. (2001). Combination of decompressive craniectomy and mild hypothermia ameliorates infarction volume after permanent focal ischemia in rats. Stroke 32, 2675-2681. doi: 10.1161/hs1101.098369 
Fann, D. Y. W., Lee, S. Y., Manzanero, S., Chunduri, P., Sobey, C. G., and Arumugam, T. V. (2013a). Pathogenesis of acute stroke and the role of inflammasomes. Ageing Res. Rev. 12, 941-966. doi: 10.1016/j.arr.2013. 09.004

Fann, D. Y. W., Lee, S. Y., Manzanero, S., Tang, S. C., Gelderblom, M., Chunduri, P., et al. (2013b). Intravenous immunoglobulin suppresses NLRP1 and NLRP3 inflammasome-mediated neuronal death in ischemic stroke. Cell Death Dis. 4:e790. doi: 10.1038/cddis.2013.326

Fisher, M., and Saver, J. L. (2015). Future directions of acute ischaemic stroke therapy. Lancet Neurol. 14, 758-767. doi: 10.1016/s1474-4422(15)00054-x

Gasior, M., Rogawski, M. A., and Hartman, A. L. (2006). Neuroprotective and disease-modifying effects of the ketogenic diet. Behav. Pharmacol. 17, 431-439. doi: 10.1097/00008877-200609000-00009

Gibson, C. L., Murphy, A. N., and Murphy, S. P. (2012). Stroke outcome in the ketogenic state-a systematic review of the animal data. J. Neurochem. 123, 52-57. doi: 10.1111/j.1471-4159.2012.07943.x

Gross, O., Thomas, C. J., Guarda, G., and Tschopp, J. (2011). The inflammasome: an integrated view. Immunol. Rev. 243, 136-151. doi: 10.1111/j.1600-065x.2011. 01046.x

Hartman, A. L., Gasior, M., Vining, E. P. G., and Rogawski, M. A. (2007). The neuropharmacology of the ketogenic diet. Pediatr. Neurol. 36, 281-292. doi: 10.1016/j.pediatrneurol.2007.02.008

Jo, E. K., Kim, J. K., Shin, D. M., and Sasakawa, C. (2016). Molecular mechanisms regulating NLRP3 inflammasome activation. Cell. Mol. Immunol. 13, 148-159. doi: $10.1038 / \mathrm{cmi} .2015 .95$

Kawabori, M., and Yenari, M. A. (2015). Inflammatory responses in brain ischemia. Curr. Med. Chem. 22, 1258-1277. doi: 10.2174/0929867322666150 209154036

Knott, A. B., Perkins, G., Schwarzenbacher, R., and Bossy-Wetzel, E. (2008). Mitochondrial fragmentation in neurodegeneration. Nat. Rev. Neurosci. 9, 505-518. doi: 10.1038/nrn2417

Lerner, A. G., Upton, J. P., Praveen, P. V., Ghosh, R., Nakagawa, Y., Igbaria, A., et al. (2012). IRE1 $\alpha$ induces thioredoxin-interacting protein to activate the NLRP3 inflammasome and promote programmed cell death under irremediable ER stress. Cell Metab. 16, 250-264. doi: 10.1016/j.cmet.2012. 07.007

McEwen, B. R., and Paterson, P. G. (2010). Caloric restriction provided after global ischemia does not reduce hippocampal cornu ammonis injury or improve functional recovery. Neuroscience 166, 263-270. doi: 10.1016/j.neuroscience. 2009.11.076

Meckling, K. A., Gauthier, M., Grubb, R., and Sanford, J. (2002). Effects of a hypocaloric, low-carbohydrate diet on weight loss, blood lipids, blood pressure, glucose tolerance, and body composition in free-living overweight women. Can. J. Physiol. Pharmacol. 80, 1095-1105. doi: 10.1139/y02-140

Menu, P., Mayor, A., Zhou, R., Tardivel, A., Ichijo, H., Mori, K., et al. (2012). ER stress activates the NLRP3 inflammasome via an UPR-independent pathway. Cell Death Dis. 3:e261. doi: 10.1038/cddis.2011.132

Minutoli, L., Puzzolo, D., Rinaldi, M., Irrera, N., Marini, H., Arcoraci, V., et al. (2016). ROS-mediated NLRP3 inflammasome activation in brain, heart, kidney, and testis ischemia/reperfusion injury. Oxid. Med. Cell. Longev. 2016:2183026. doi: 10.1155/2016/2183026

Offermanns, S., and Schwaninger, M. (2015). Nutritional or pharmacological activation of $\mathrm{HCA}_{2}$ ameliorates neuroinflammation. Trends Mol. Med. 21, 245-255. doi: 10.1016/j.molmed.2015.02.002

Ogura, Y., Sutterwala, F. S., and Flavell, R. A. (2006). The inflammasome: first line of the immune response to cell stress. Cell 126, 659-662. doi: 10.1016/j.cell. 2006.08.002

Oslowski, C. M., Hara, T., O’Sullivan-Murphy, B., Kanekura, K., Lu, S. M., Hara, M., et al. (2012). Thioredoxin-interacting protein mediates ER stressinduced $\beta$ cell death through initiation of the inflammasome. Cell Metab. 16, 265-273. doi: 10.1016/j.cmet.2012.07.005

Otera, H., and Mihara, K. (2012). Mitochondrial dynamics: functional link with apoptosis. Int. J. Cell. Biol. 2012:821676. doi: 10.1155/2012/821676

Prins, M. L. (2008). Cerebral metabolic adaptation and ketone metabolism after brain injury. J. Cereb. Blood Flow Metab. 28, 1-16. doi: 10.1038/sj.jcbfm. 9600543

Puchowicz, M. A., Zechel, J. L., Valerio, J., Emancipator, D. S., Xu, K., Pundik, S., et al. (2008). Neuroprotection in diet-induced ketotic rat brain after focal ischemia. J. Cereb. Blood Flow Metab. 28, 1907-1916. doi: 10.1038/jcbfm. 2008.79

Rahman, M., Muhammad, S., Khan, M. A., Chen, H., Ridder, D. A., MüllerFielitz, H., et al. (2014). The $\beta$-hydroxybutyrate receptor HCA2 activates a neuroprotective subset of macrophages. Nat. Commun. 5:3944. doi: 10.1038/ncomms4944

Rayamajhi, M., and Miao, E. A. (2014). The RIP1-RIP3 complex initiates mitochondrial fission to fuel NLRP3. Nat. Immunol. 15, 1100-1102. doi: 10.1038/ni.3030

Roussel, B. D., Kruppa, A. J., Miranda, E., Crowther, D. C., Lomas, D. A., and Marciniak, S. J. (2013). Endoplasmic reticulum dysfunction in neurological disease. Lancet Neurol. 12, 105-118. doi: 10.1016/S1474-4422(12)70238-7

Seifert, H. A., and Pennypacker, K. R. (2014). Molecular and cellular immune responses to ischemic brain injury. Transl. Stroke Res. 5, 543-553. doi: 10.1007/s12975-014-0349-7

Shaafi, S., Mahmoudi, J., Pashapour, A., Farhoudi, M., Sadigh-Eteghad, S., and Akbari, H. (2014). Ketogenic diet provides neuroprotective effects against ischemic stroke neuronal damages. Adv. Pharm. Bull. 4, 479-481. doi: $10.5681 / \mathrm{apb} .2014 .071$

Shai, I., Schwarzfuchs, D., Henkin, Y., Shahar, D. R., Witkow, S., Greenberg, I., et al. (2008). Weight loss with a low-carbohydrate, Mediterranean, or low-fat diet. N. Engl. J. Med. 359, 229-241. doi: 10.1056/NEJMoa0708681

Sharman, M. J., Kraemer, W. J., Love, D. M., Avery, N. G., Gómez, A. L., Scheett, T. P., et al. (2002). A ketogenic diet favorably affects serum biomarkers for cardiovascular disease in normal-weight men. J. Nutr. 132, 1879-1885. doi: 10.1093/jn/132.7.1879

Smirnova, E., Griparic, L., Shurland, D. L., and van der Bliek, A. M. (2001) Dynamin-related protein Drp1 is required for mitochondrial division in mammalian cells. Mol. Biol. Cell 12, 2245-2256. doi: 10.1091/mbc.12.8.2245

Su, S. W., Cilio, M. R., Sogawa, Y., Silveira, D. C., Holmes, G. L., and Stafstrom, C. E. (2000). Timing of ketogenic diet initiation in an experimental epilepsy model. Dev. Brain Res. 125, 131-138. doi: 10.1016/S01653806(00)00130-9

Suzuki, M., Suzuki, M., Kitamura, Y., Mori, S., Sato, K., Dohi, S., et al. (2002). $\beta$-hydroxybutyrate, a cerebral function improving agent, protects rat brain against ischemic damage caused by permanent and transient focal cerebral ischemia. Jpn. J. Pharmacol. 89, 36-43. doi: 10.1254/jjp.89.36

Suzuki, M., Suzuki, M., Sato, K., Dohi, S., Sato, T., Matsuura, A., et al. (2001). Effect of $\beta$-hydroxybutyrate, a cerebral function improving agent, on cerebral hypoxia, anoxia and ischemia in mice and rats. Jpn. J. Pharmacol. 87, 143-150. doi: $10.1254 /$ jjp. 87.143

Tai, K. K., Nguyen, N., Pham, L., and Truong, D. D. (2008). Ketogenic diet prevents cardiac arrest-induced cerebral ischemic neurodegeneration. J. Neural Transm. Vienna 115, 1011-1017. doi: 10.1007/s00702-008-0050-7

Thio, L. L., Erbayat-Altay, E., Rensing, N., and Yamada, K. A. (2006). Leptin contributes to slower weight gain in juvenile rodents on a ketogenic diet. Pediatr. Res. 60, 413-417. doi: 10.1203/01.pdr.0000238244. 54610.27

Tobin, M. K., Bonds, J. A., Minshall, R. D., Pelligrino, D. A., Testai, F. D., and Lazarov, O. (2014). Neurogenesis and inflammation after ischemic stroke: what is known and where we go from here. J. Cereb. Blood Flow Metab. 34, 1573-1584. doi: 10.1038/jcbfm.2014.130

Tschopp, J., and Schroder, K. (2010). NLRP3 inflammasome activation: the convergence of multiple signalling pathways on ROS production? Nat. Rev. Immunol. 10, 210-215. doi: 10.1038/nri2725

Volek, J. S., Phinney, S. D., Forsythe, C. E., Quann, E. E., Wood, R. J., Puglisi, M. J., et al. (2009). Carbohydrate restriction has a more favorable impact on the metabolic syndrome than a low fat diet. Lipids 44, 297-309. doi: 10.1007/s11745-008-3274-2

Volek, J. S., Sharman, M. J., and Forsythe, C. E. (2005). Modification of lipoproteins by very low-carbohydrate diets. J. Nutr. 135, 1339-1342. doi: $10.1093 / \mathrm{jn} / 135.6 .1339$

Westermann, B. (2002). Merging mitochondria matters: cellular role and molecular machinery of mitochondrial fusion. EMBO Rep. 3, 527-531. doi: 10.1093/embo-reports/kvf113

Xin, Q., Ji, B., Cheng, B., Wang, C., Liu, H., Chen, X., et al. (2014). Endoplasmic reticulum stress in cerebral ischemia. Neurochem. Int. 68, 18-27. doi: 10.1016/j. neuint.2014.02.001 
Yang, Q., Guo, M., Wang, X., Zhao, Y., Zhao, Q., Ding, H., et al. (2017). Ischemic preconditioning with a ketogenic diet improves brain ischemic tolerance through increased extracellular adenosine levels and hypoxiainducible factors. Brain Res. 1667, 11-18. doi: 10.1016/j.brainres.2017. 04.010

Yang, F., Wang, Z., Wei, X., Han, H., Meng, X., Zhang, Y., et al. (2014). NLRP3 deficiency ameliorates neurovascular damage in experimental ischemic stroke. J. Cereb. Blood Flow Metab. 34, 660-667. doi: 10.1038/jcbfm. 2013.242

Yoon, J. S., Mughal, M. R., and Mattson, M. P. (2011). Energy restriction negates NMDA receptor antagonist efficacy in ischemic stroke. Neuromolecular. Med. 13, 175-178. doi: 10.1007/s12017-011-8145-y

Youm, Y. H., Nguyen, K. Y., Grant, R. W., Goldberg, E. L., Bodogai, M., Kim, D., et al. (2015). The ketone metabolite $\beta$-hydroxybutyrate blocks NLRP3 inflammasome-mediated inflammatory disease. Nat. Med. 21, 263-269. doi: 10.1038/nm.3804

Zhao, Y., Chen, F., Chen, S., Liu, X., Cui, M., and Dong, Q. (2013). The Parkinson's disease-associated gene PINK1 protects neurons from ischemic damage by decreasing mitochondrial translocation of the fission promoter Drp1. J. Neurochem. 127, 711-722. doi: 10.1111/jnc.12340

Zhou, R., Yazdi, A. S., Menu, P., and Tschopp, J. (2011). A role for mitochondria in NLRP3 inflammasome activation. Nature 469, 221-225. doi: $10.1038 /$ nature09663

Conflict of Interest Statement: The authors declare that the research was conducted in the absence of any commercial or financial relationships that could be construed as a potential conflict of interest.

Copyright (c) 2018 Guo, Wang, Zhao, Yang, Ding, Dong, Chen and Cui. This is an open-access article distributed under the terms of the Creative Commons Attribution License (CC BY). The use, distribution or reproduction in other forums is permitted, provided the original author(s) and the copyright owner are credited and that the original publication in this journal is cited, in accordance with accepted academic practice. No use, distribution or reproduction is permitted which does not comply with these terms. 